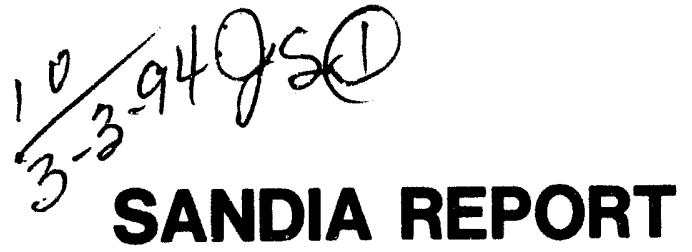

SAND94-0139 $\cdot$ UC-906

Unlimited Release

Printed January 1994

⿶

\title{
- A Model for Forming Airborne Synthetic Aperture Radar Images of Underground Targets
}

Armin W. Doerry

Prepared by

Sandia National Laboratorles

Albuquerque, Now Moxico 87185 and Livermore, Callifornia 94580

for the United 8tatos Dopertment of Energy

under Contract DE-ACO4-OAAL85000 
Issued by Sandia National Laboratories, operated for the United States Department of Energy by Sandia Corporation.

NOTICE: This report was prepared as an account of work sponsored by an agency of the United States Government. Neither the United States Government nor any agency thereof, nor any of their employees, nor any of their contractors, subcontractors, or their employees, makes any warranty, express or implied, or assumes any legal liability or responsibility for the accuracy, completeness, or usefulness of any information, apparatus, product, or process disclosed, or represents that its use would not infringe privately owned rights. Reference herein to any specific commercial product, process, or service by trade name, trademark, manufacturer, or otherwise, does not necessarily constitute or imply its endorsement, recommendation, or favoring by the United States Government, any agency thereof or any of their contractors or subcontractors. The views and opinions expressed herein do not necessarily state or reflect those of the United States Government, any agency thereof or any of their contractors.

Printed in the United States of America. This report has been reproduced directly from the best available copy.

Available to DOE and DOE contractors from Office of Scientific and Technical Information PO Box 62

Oak Ridge, TN 37831

Prices available from (615) 576-8401, FTS $626-8401$

Available to the public from

National Technical Information Service

US Department of Commerce

5285 Port Royal Rd

Springfield, VA 22161

NTIS price codes

Printed copy: A04

Microfiche copy: A01 
SAND94-0139

Distribution

Unlimited Release

Category

Printed January 1994

UC-906

\title{
A Model for Forming Airborne Synthetic Aperture Radar Images of Underground Targets
}

\author{
Armir W. Doerry \\ Synthetic Aperture Radar Department, 2345 \\ Sandia National Laboraiories \\ Albuquerque, NM 87185-0529
}

\begin{abstract}
Synthetic Apertיre Radar (SAR) from an airborne platform has been proposed for imaging targets beneath the earth's surface. The propagation of the radar's energy within the ground, however, is much different than in the earth's atmosphere. The result is signal refraction, echo delay, propagation losses, dispersion, and volumetric scattering. These all combine to make SAR image formation from an airborne platform much more challenging than a surface imaging counterpart. This report treats the ground as a lossy dispersive half-space, and presents a model for the radar echo based on measurable parameters. The model is then used to explore various imaging schemes, and image properties. Dynamic range is discussed, as is the inpact of loss on dynamic range. Modified window functions are proposed to mitigate effects of sidelobes of shallow targets overwhelming deeper targets.
\end{abstract}

\section{MASTER}

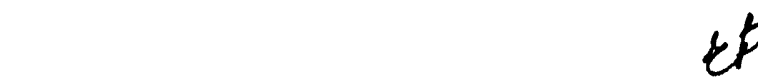




\section{ACKNOWLEDGEMENTS}

This report represents work undertaken under a Sandia National Laboratories (SNL) Laboratory Directed Research and Development (LDRD) program. The program manager is Dan Cress (9131) and the technical project leader is Bill Boverie (9135).

While this program has a number of participants, all of whom have influenced the direction of study, the author wishes to thank Billy Brock (2343) and Tom Cordaro (2344) in particular, for often lending their expertise and opinion to the subjects developed here within.

This work performed at Sandia National Laboratories is supported by the U.S. Department of Energy under contract DE-AC04-94AL85000. 


\section{TABLE OF CONTENTS}

ABSTRACT …

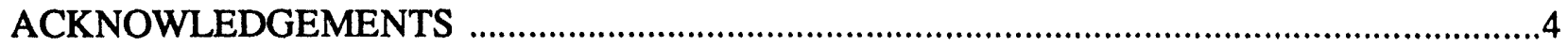

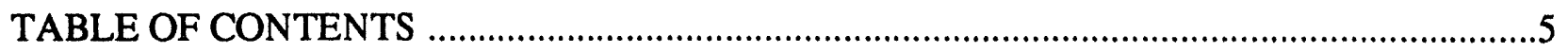

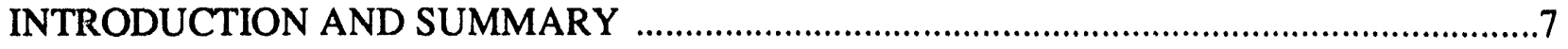

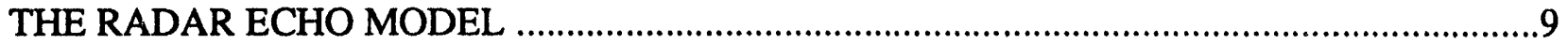

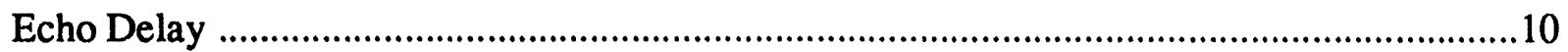

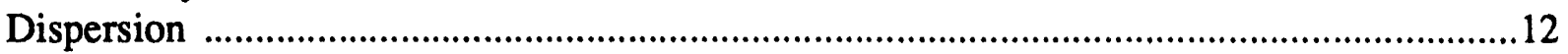

Attenuation and Power Loss within the Dielectric ...........................................................15

Soil Types and their Properties ......................................................................................

AN AIRBORNE SYNTHETIC APERTURE RADAR PHASE HISTORY MODEL ................19

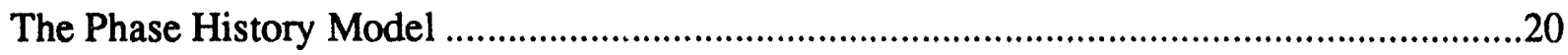

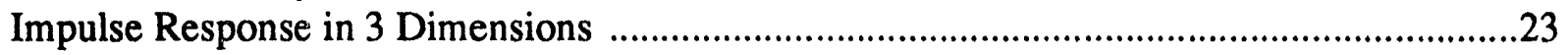

Some Collection Geometry Examples ..........................................................................23

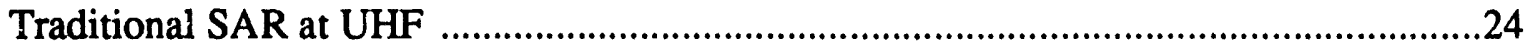

SAR at UHF with Multiple Apertures .......................................................................25

Downward Looking SAR at UHF with Multiple Apertures ...............................................26

Narrow Band Downward Looking SAR at UHF .........................................................27

Downward Looking SAR at UHF with Multiple Apertures (Shallow Target) .................28

Downward Looking SAR at VHF .............................................................................29

Some Comments About the Previous Examples .................................................................30

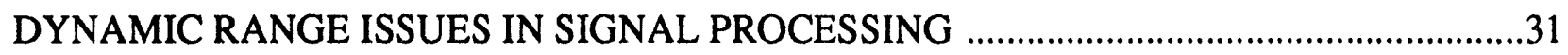

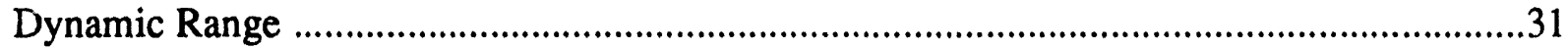

Loss Normalized Depth Impulse Response - Matched Filter Processing ................................32

Loss Normalized Depth Impulse Response - DFT Processing ……………...............................36

Loss Compensated Window Functions for Near Sidelobe Suppression ...................................39

Synthesizing Loss Compensated Windows .............................................................................42

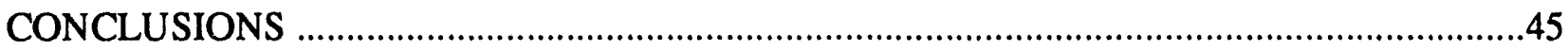

APPENDIX A - DERIVATION OF RADAR ECHO MODEL ………….................................47

APPENDIX B - DERIVATION OF OPTIMAL COMPLEX FILTER …………………............49

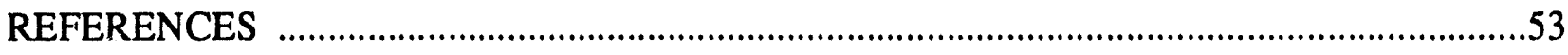

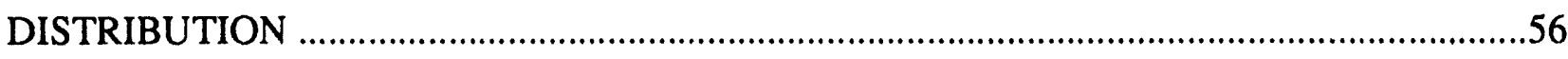


THIS PAGE INTENTIONALLY BLANK

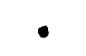




\section{INTRODUCTION AND SUMMARY}

There is significant interest in detecting, locating, and identifying 'aargets' beneath the earth's surface. Applications span the areas of national defense, environmental monitoring, arms control and nonproliferation, geologic and mining exploration, and archeology. There is a healthy industry today of ground-based vehicle- or hand-towed ground penetrating radars (GPRs). The challenge is to cover more ground, deeper, quicker, and with better resolution. To this end, experimental systems are emerging using aircraft based, airborne ground penetrating radars. A problem arises that resolution falls off with distance (altitude), and a strong surface return interferes with the data. This has led to the consideration of synthetic aperture radar (SAR) techniques to regain resolution and surface return mitigation. To date, however, most SAR efforts seem to apply surface imaging techniques to the subsurface imaging problem. Very little has been written in the literature thus far dealing with problems unique to imaging underground targets with an airborne SAR. The impact of signal refraction, accurate echo delay, propagation losses, dispersion, and volumetric scattering on SAR data seem to have been largely ignored in a quantitative manner. This report attempts to fill some of this information void.

Within this report is presented first a model for radar echo delay time from an airborne radar to a subsurface target that takes into account signal refraction and dispersion. Later, propagation losses are also included. Dispersion is discussed and its effect on wideband signals. Then SAR imaging is addressed with models presented. These models are then used to illustrate effects of some different imaging geometries. Dynamic range for imaging buried targets is discussed and the concept of loss normalized depth impulse response is presented. It is shown how loss can interfere with dynamic range via scatterer sidelobes. Finally, a mitigation method for this interference is presented.

To summarize the results so far, it seems entirely possible to image targets buried below the earth's surface from an airborne SAR, with some qualifiers. First, penetration depth will depend very much on soil dielectric properties, and range from centimeters to many meters. At UHF frequencies we are likely to be able to penetrate a few meters in a large number of soils, with at least 1 meter resolution or better. Lower frequencies will penetrate more, but with worse resolution. Focussing SAR images will need to take into account soil parameters. Errors in measured or assumed propagation parameters will manifest themselves in target position estimate inaccuracies, and image defocussing. Imaging with a 2-dimensional spatial aperture as well as a frequency aperture is extremely desirable to localize a target's 3-dimensional impulse response. However, coherency is required for all spatial sample points, to properly focus the image. This could be a tough problem for motion measurement systems. Throughout this report are presented examples to illustrate the concepts discussed. 
THIS PAGE INTENTIONALLY BLANK

$-8-$ 


\section{THE RADAR ECHO MODEL}

Consider a dielectric boundary, above which is free space, and below which is a homogeneous, lossy, and dispersive medium. Let a cartesian coordinate system be chosen with the following properties.

The $x-y$ plane is the dielectric boundary with free space in the $+z$ direction.

The dielectric has complex relative permittivity $\varepsilon$, and complex relative permeability $\mu$. The conductivity is included in $\varepsilon$. Both $\varepsilon$ and $\mu$ are generally functions of frequency.

The origin of this coordinate system is identified as the Central Reference Point (CRP).

Within this coordinate system we create the following, as in figure 1.

We place a target at location $(0,0,-d)$, directly beneath the CRP within the dielectric.

We place a radar sensor with phase center at location $\left(\mathrm{x}_{0}, \mathrm{y}_{0}, \mathrm{z}_{0}\right)$, in free space. A vector from the CRP to this point is defined as $\mathbf{r}_{c}$. The angle between $\mathbf{r}_{c}$ and the $\mathrm{x}$ $y$ plane is defined as the depression angle $\psi$.

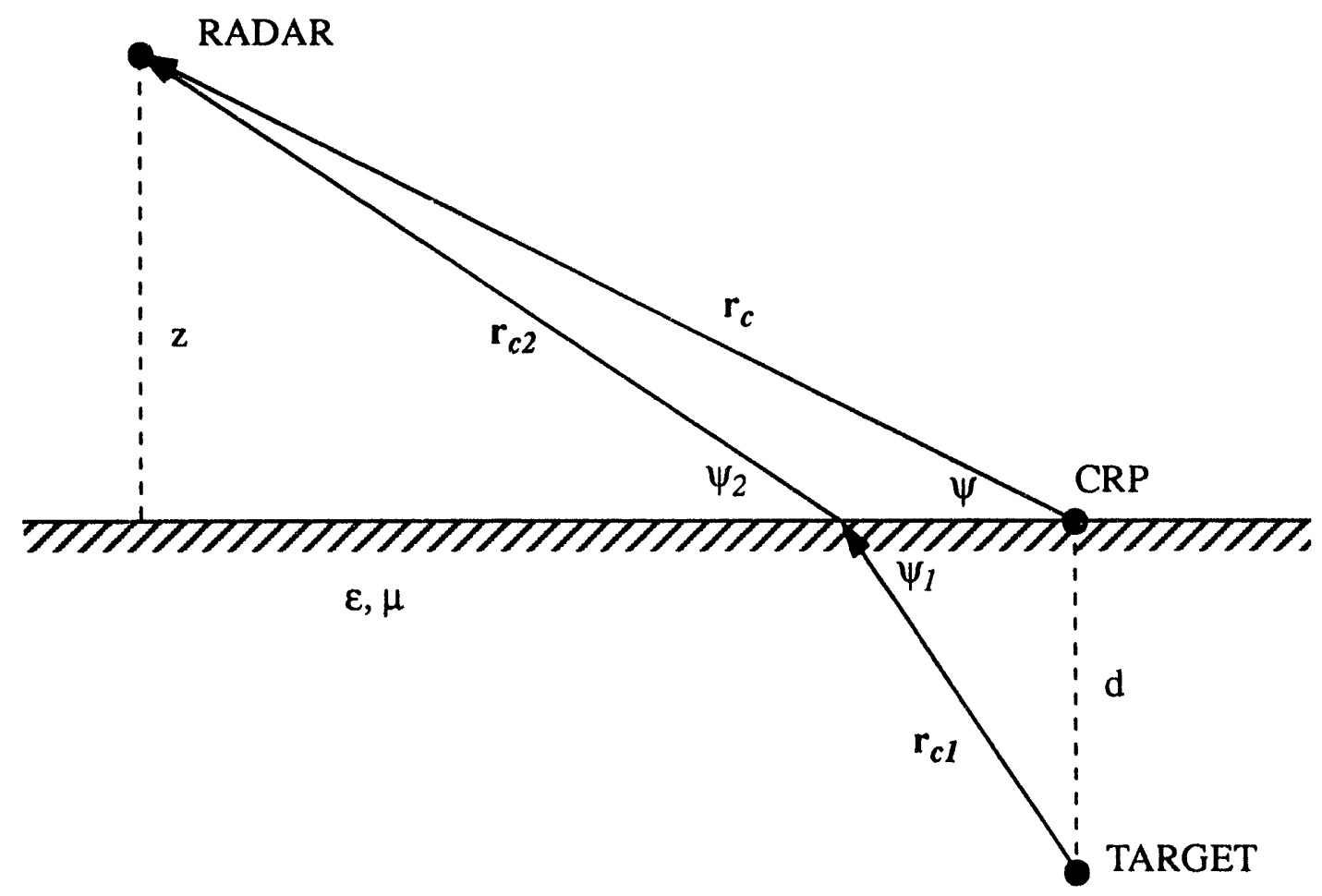

Figure 1. Geometry of Radar Echo 
If the radar emits energy that reflects from the target, the echo will follow the ray path identified by vectors $r_{c 1}$ and $r_{c 2}$, which make angles with the $x-y$ plane of $\psi_{1}$ and $\psi_{2}$ respectively.

\subsection{Echo Delay}

The echo delay time is then given by

$$
\tau_{c}=\frac{2\left\|r_{c 1}\right\|}{c_{1}}+\frac{2\left\|r_{c 2}\right\|}{c}
$$

where

$$
\begin{aligned}
\tau_{c} & =\text { radar echo delay time, } \\
c & =\text { velocity of light in free space, and } \\
c_{1} & =\text { velocity of propagation in the lossy half space. }
\end{aligned}
$$

Imaging using (1) is discussed by $\mathrm{Xu}, \mathrm{CaO}$, and $\mathrm{Su}$ [15]. The problem is that for a lossy medium, even if $\varepsilon$ and $\mu$ are known, given only $r_{c}$ and $d$, exact values for $\left\|r_{c 1}\right\|$ and $\left\|r_{c 2}\right\|$ are generally difficult to compute.

However, by making the plausible assumption for an airborne $\operatorname{SAR}$ that $\psi_{2} \approx \psi$, we can find a closed form approximate solution for the echo delay time as

$$
\tau_{c} \approx \frac{2}{c}\left(\left\|r_{c}\right\|+\operatorname{Real}\left[d \eta \sqrt{1-\left(\frac{\cos \psi}{\eta}\right)^{2}}\right]\right),
$$

where

$$
\begin{aligned}
\eta & =\text { complex refraction index for the lossy dielectric }=\sqrt{\varepsilon \mu}=\eta_{R}+j \eta_{l}, \\
\eta_{R} & =\operatorname{Real}[\eta], \text { and } \\
\eta_{I} & =\operatorname{Imag}[\eta] .
\end{aligned}
$$

The derivation for (2) is given in Appendix A.

If $\left(\frac{\cos \psi}{\eta}\right)^{2} \ll 1$, then the further approximation may be made

$$
\tau_{c} \approx \frac{2}{c}\left(\left\|r_{c}\right\|+d \eta_{R}\left(1-\frac{\cos ^{2} \psi}{2\|\eta\|^{2}}\right)\right),
$$

The grossest approximation ignores depression angle effects, and is given by

$$
\tau_{c} \approx \frac{2}{c}\left(\left\|r_{c}\right\|+d \eta_{R}\right) .
$$


Which approximation to use depends largely on the accuracy required, which in turn depends on the circumstances of its use. As an illustration, consider the following example.

Example 1. Consider the following parameters

$$
\begin{array}{ll}
\left\|r_{c}\right\|=500 \mathrm{~m}, & \\
d=3 \mathrm{~m}, & \\
\varepsilon=4.5-\mathrm{j} 1 \quad & \begin{array}{l}
\text { (representative of San Antonio Clay Loam with } 5 \% \\
\text { water content at } 300 \mathrm{MHz} . \text { See reference [2]), }
\end{array} \\
\mu=1, &
\end{array}
$$$$
\text { water content at } 300 \mathrm{MHz} \text {. See reference [2]), }
$$

and let us define effective range as the equivalent range in free space to yield a particular echo delay time, that is

$$
R_{\text {eff }} \equiv \frac{c}{2} \tau_{c}
$$

The effective range measure is useful in that it allows us to measure distances against transmitted wavelengths in free space.

The various approximations are then plotted against an exact numerical solution in terms of effective range in figure 2 .

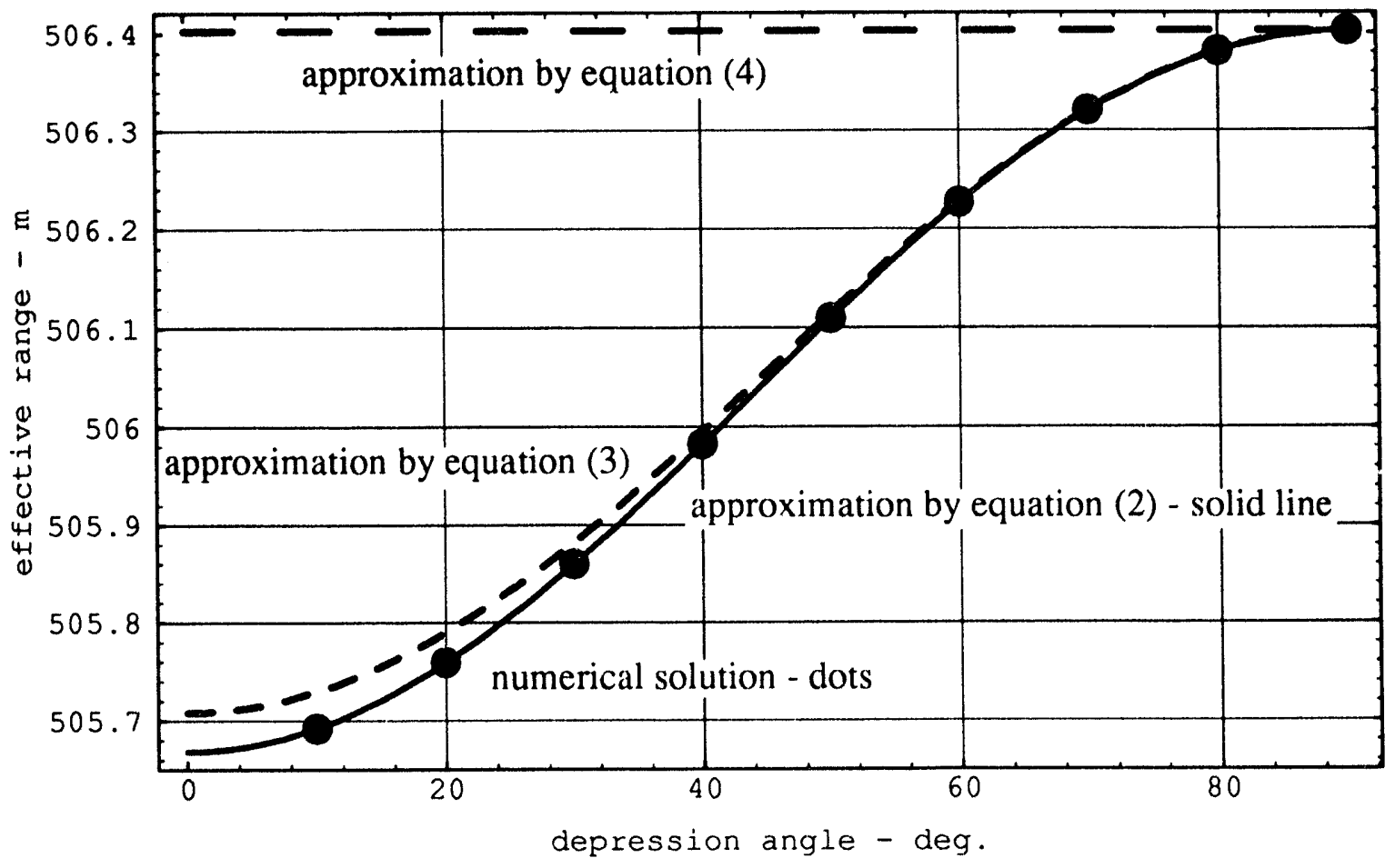

Figure 2. Comparison of various approximations in terms of effective range. 
For this example we may make several observations.

- The approximation by equation (2) is nearly error free. In fact, the approximation by equation (2) meets the numerical solution to within $5 \times 10^{-4} \mathrm{~m}$.

- The approximations by equations (3) and (4) improve with steeper depression angles.

- The approximation by equation (3) is in error by less than $0.04 \mathrm{~m}$, which is still adequate for coherent imaging at $300 \mathrm{MHz}$, over any range of depression angles.

- The approximation by equation (4) shows no depression angle dependence, and is useful for coherent imaging at $300 \mathrm{MHz}$ only over very narrow ranges of depression angles. Even then, at all but vertical depression angles, there will be a time offset (or equivalently an effective range offset).

\subsection{Dispersion}

Consider the approximation by equation (2) as a reasonable model. We repeat it here.

$$
\tau_{c} \approx \frac{2}{c}\left(\left\|r_{c}\right\|+\operatorname{Real}\left[d \eta \sqrt{1-\left(\frac{\cos \psi}{\eta}\right)^{2}}\right]\right)
$$

In general, $\eta$ is a function of frequency, $\omega$, therefor $\tau_{c}$ is also a function of frequency. This effect is identified as dispersion, and can be a nuisance when dealing with wideband signals, such as very short pulses (actual or synthetic). From equation (2) we see that dispersion is magnified linearly with depth, $d$. Therefor it may not be a problem with shallow targets, but gets progressively worse with deeper targets, as one might expect. We illustrate dispersion with the following example, borrowing data from Hipp [7] via the model of Brock and Patitz [2].

Example 2. Consider the following parameters (essentially the same as example 1).

$$
\begin{aligned}
& \left\|r_{c}\right\|=500 \mathrm{~m}, \\
& \psi=60 \mathrm{deg} . \\
& d=\quad 3 \mathrm{~m}, \\
& \varepsilon=\quad \begin{array}{l}
\text { representative of San Antonio Clay Loam with } 5 \% \text { water content } \\
\text { from reference [2], }
\end{array} \\
& \mu=1,
\end{aligned}
$$

FIgure 3 repeats the dielectric constant curve from reference [2]. Figure 4 shows the complex refraction index calculated from this curve and $\mu$. Finally, figure 5 shows the effective range for the various frequencies in the range $30 \mathrm{MHz}$ to 1 $\mathrm{GHz}$. 


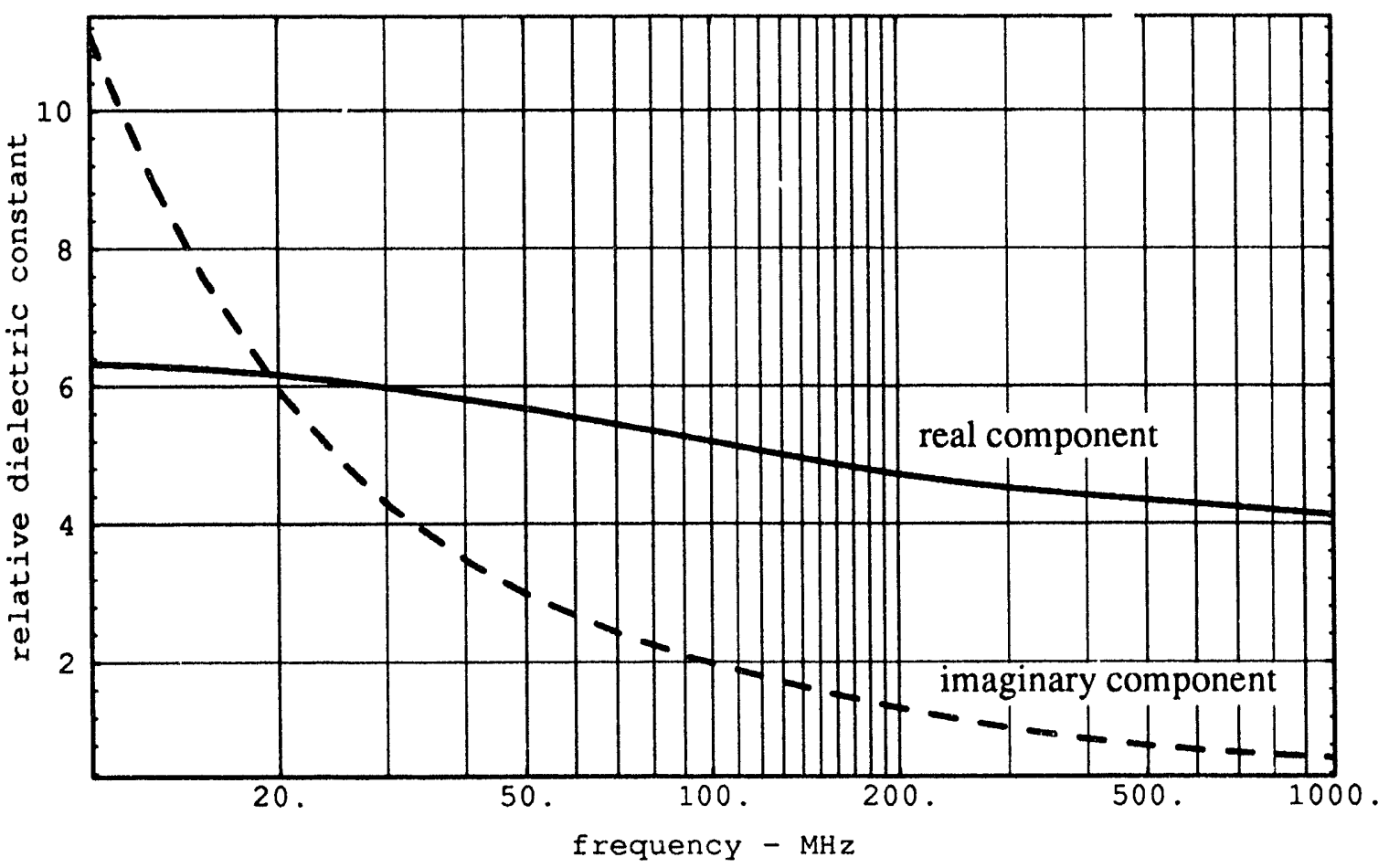

Figure 3. Dielectric Constant for San Antonio Clay Loam with 5\% Water.

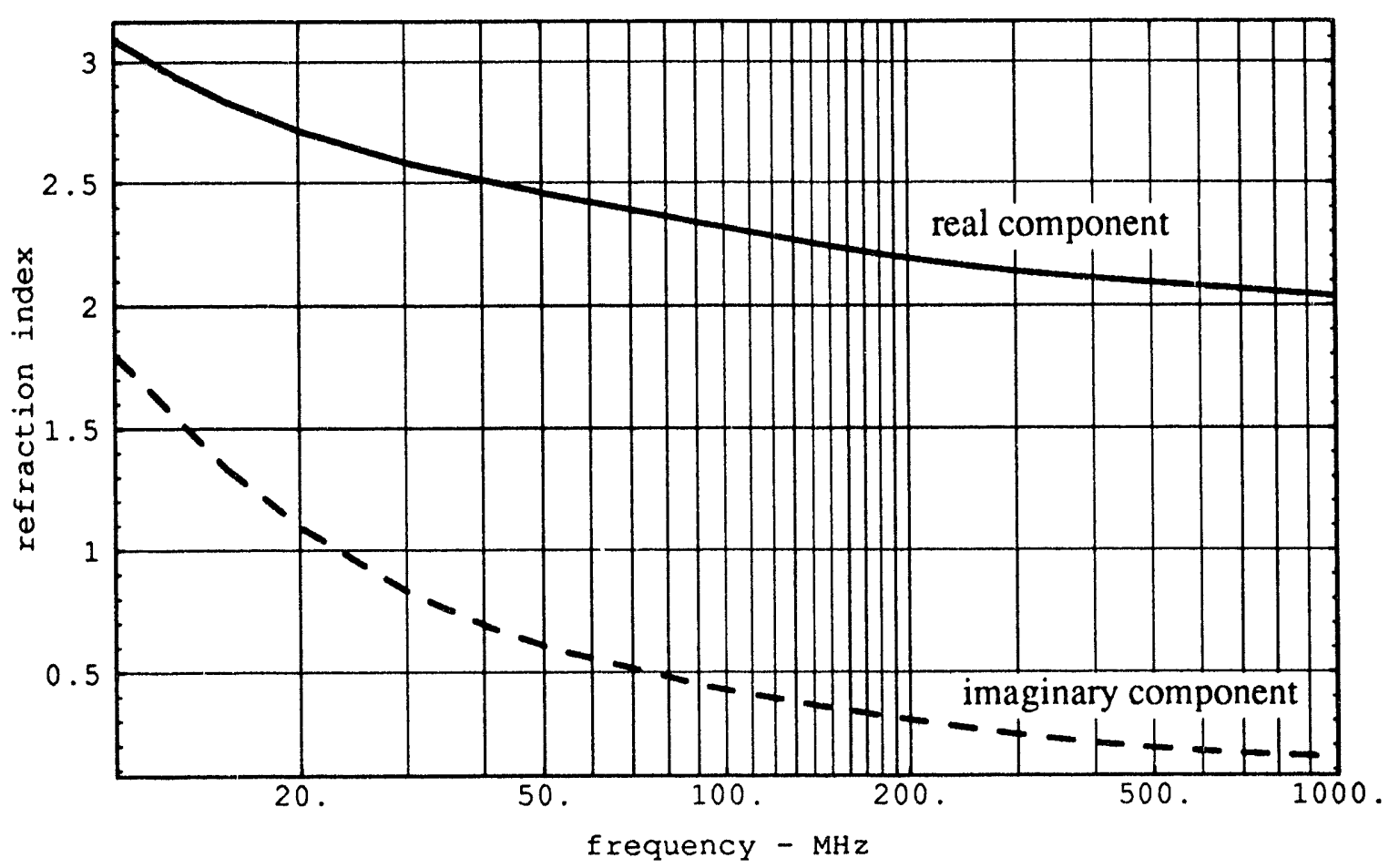

Figure 4. Refraction Index for San Antonio Clay Loam with 5\% Water. 


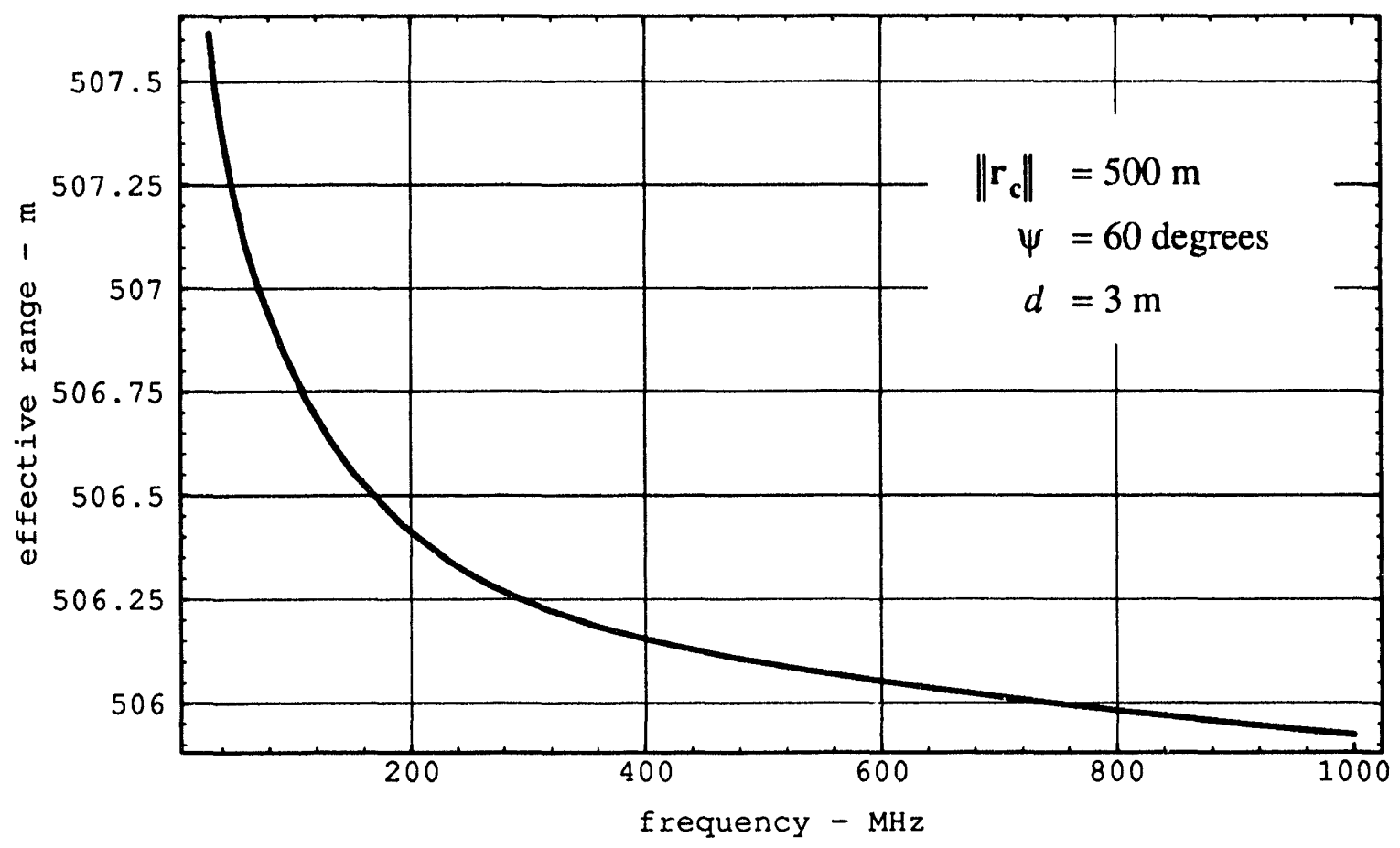

Figure 5. Effective Range vs. Frequency for San Antonio Clay Loam with 5\% Water.

For this example we make the following observations.

- Targets at other depths would show the same basic curve shape as in figure 5, but with different scaling on the vertical axis. That is, at a depth of $12 \mathrm{~m}$, the dispersion would be four times worse.

- For this soil, a finite bandwidth of say $200 \mathrm{MHz}$ at lower frequencies will exhibit more dispersion across its band than across a $200 \mathrm{MHz}$ band at higher frequencies. It is unknown at this point how applicable such a statement would be to other soil types.

- For this soil, a wideband pulse covering the entire band $30 \mathrm{MHz}$ to $1 \mathrm{GHz}$ may be capable of nearly $0.15 \mathrm{~m}$ free-space resolution, but will encounter dispersion greater than an effective $1.5 \mathrm{~m}$ in free space. This means that this pulse's ultimate resolution is limited by dispersion, unless the effects of dispersion can be mitigated. Transmitted impulses will be received as nonlinear chirps, and transmitted linear FM chirps will be received as nonlinear chirps. Dispersion mitigation is essentially a focussing problem.

- While figure 5 seems to favor higher frequencies from a dispersion standpoint, frequency dependent losses have been completely ignored, and in fact will generally favor lower frequencies. 


\subsection{Attenuation and Power Loss within the Dielectric}

In addition to the losses suffered by a surface imaging SAR, a ground penetrating SAR will encounter losses in crossing the air-ground interface, as well as in the ground itself. Losses in transmirsion across a dielectric boundary are discussed in reference [2]. By making the same assunir $\cdot \quad$ Is were made in arriving at equation (2), we can model the power attenuation within the diescuse.s

$$
G_{\eta} \approx \exp \left(\frac{4 \omega}{c} \operatorname{Imag}\left[d \eta \sqrt{1-\left(\frac{\cos \psi}{\eta}\right)^{2}}\right]\right) .
$$

This expression is derived in Appendix A.

Converting this expression into $\mathrm{dB}$ yields

$$
G_{\eta, d B} \approx \frac{40\left(\log _{10} e\right) \omega d}{c} \operatorname{Imag}\left[\eta \sqrt{1-\left(\frac{\cos \psi}{\eta}\right)^{2}}\right]
$$

We make the following observations.

- $\eta_{I}<0$, therefore $\mathrm{G}_{\eta}<1$, constituting a loss for $d>0$.

- The loss is greater (smaller $\mathrm{G}_{\eta}$ ) for larger $d$, and smaller $\psi$, which is expected since in both cases the ray path through the dielectric is greater.

- $\eta$ is generally a function of $\omega$. Even so, $\omega$ appears explicitly in equations (6) and (7), making loss a strong function of frequency.

To illustrate these observations consider the following example.

Example 3. Consider the same soil as in Example 2, namely San Antonio Clay Loam with 5\% water content from Hipp [7], as modelled by Brock and Patitz [2]. In addition, as before, let

$$
\begin{aligned}
& d=3 \mathrm{~m}, \\
& \mu=1,
\end{aligned}
$$

Figure 6 illustrates the dependence of two-way propagation loss within the dielectric at $300 \mathrm{MHz}$ for the target on depression angle. Figure 7 shows the dependence on frequency at a $60 \mathrm{deg}$. depression angle. It is important to note that these losses are with respect to an identical target buried just below the dielectric boundary, and do not consider the normal losses for a surface target, nor the transmissivity loss of transcending the dielectric boundary. 


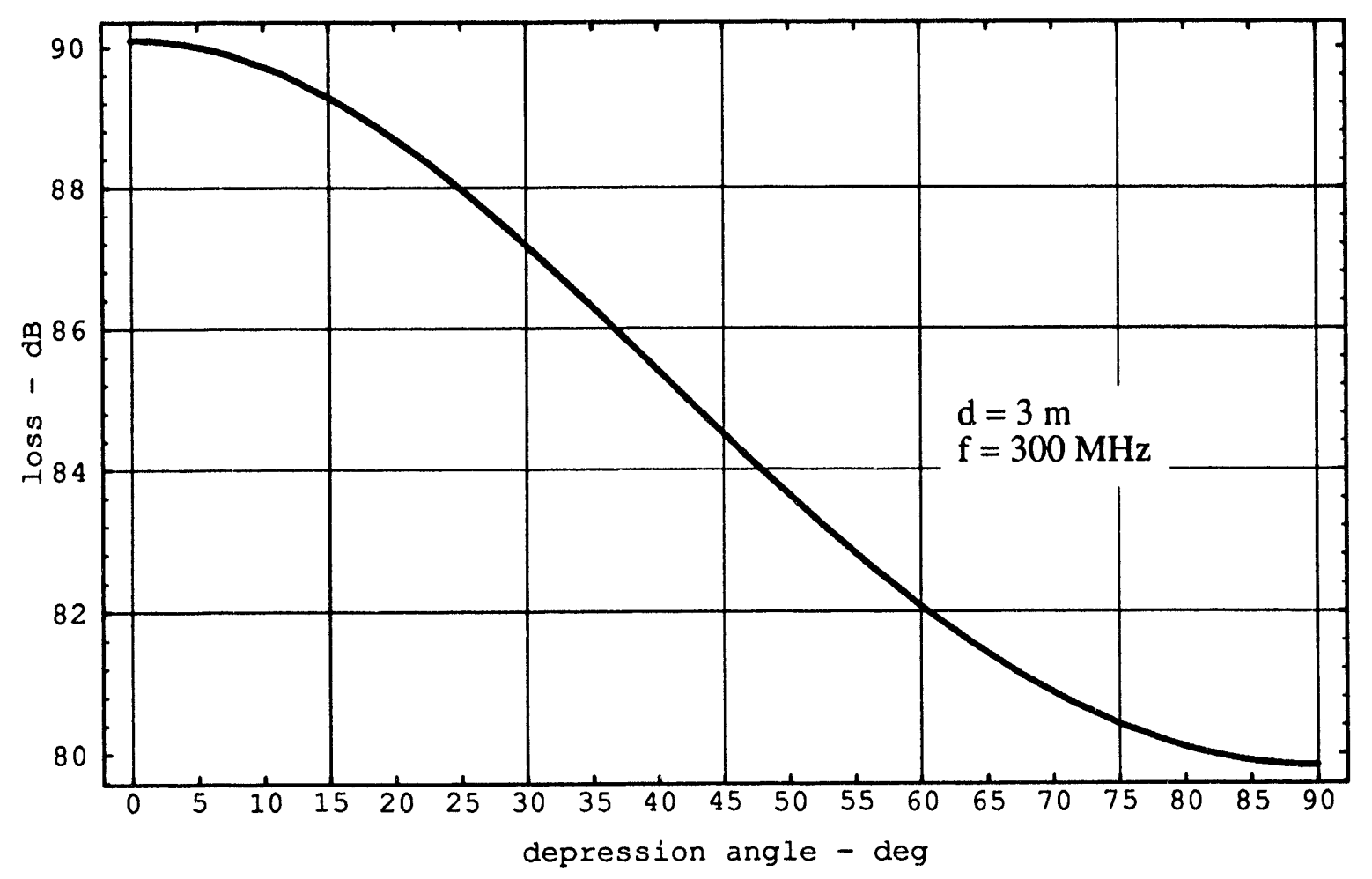

Figure 6. Two-way propagation loss as a function of depression angle.

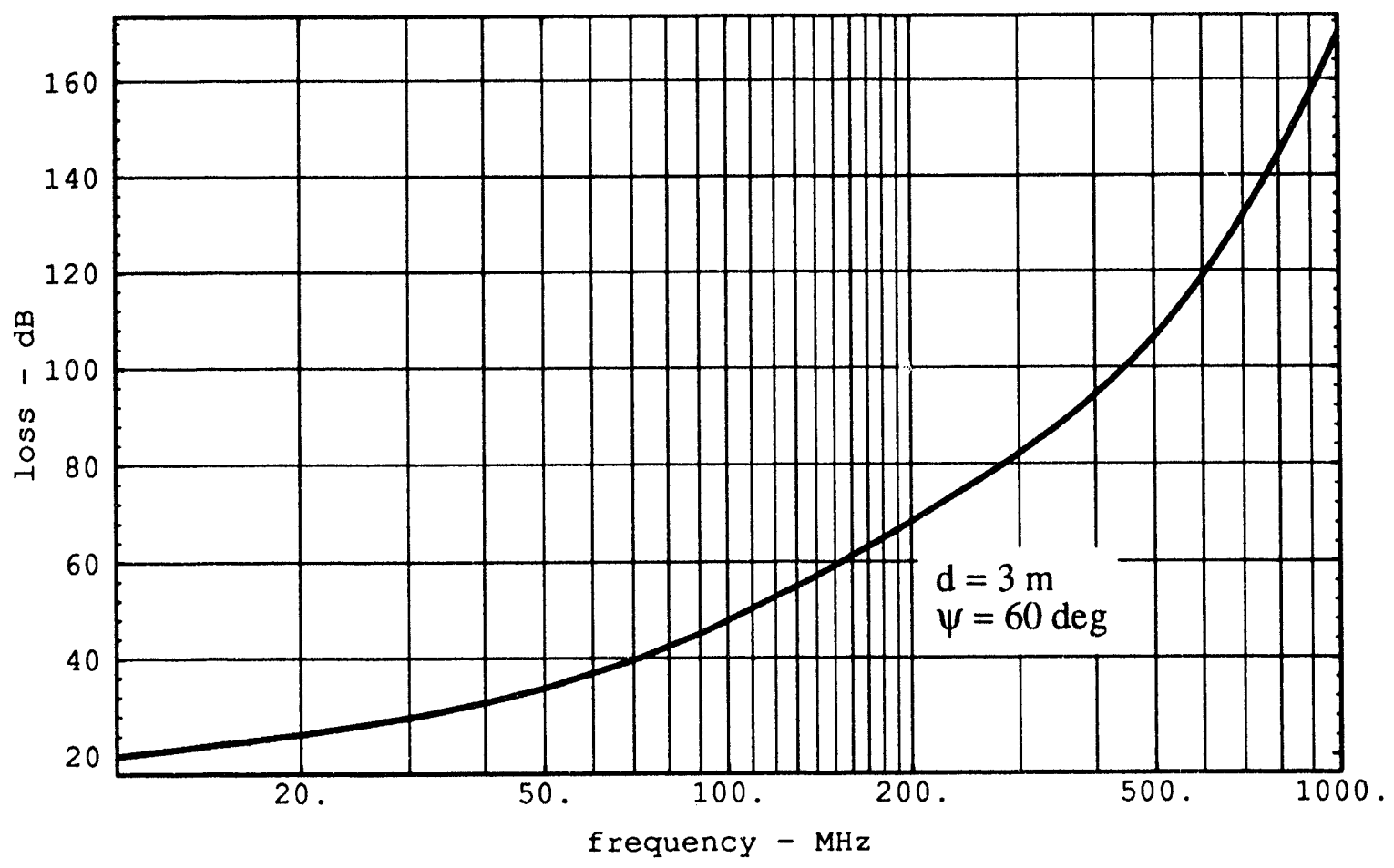

Figure 7. Two-way propagation loss as a function of frequency. 


\subsection{Soil Types and their Properties}

Soil types vary widely, and any given soil's properties will also vary widely with moisture content, compaction density, contamination, and about any other parameter you can think of. A number of other references ([2], [3], [6], [7], [8], [13], [14]) discuss this in much more detail than what will be presented here. The purpose of this section is merely to indicate the range of properties a medium may have in which a target may be buried.

Table 1 presents some data from references [2], [5], and [14], and some simple calculations of two-way propagation loss.

Table 1: Approximate Properties of Soil Types and other materials at $100 \mathrm{MHz}, 30$ degrees depression angle, target buried under 1 meter of material. (We assume $\mu=1$.)

\begin{tabular}{|c|c|c|c|c|}
\hline Material & $\begin{array}{l}\text { Approx. } \\
\text { Real(E) }\end{array}$ & $\begin{array}{c}\text { Approx. } \\
\sigma \\
(\mathrm{mho} / \mathrm{m})\end{array}$ & $\begin{array}{l}\text { Approx. } \\
\text {-Imag }(\varepsilon)\end{array}$ & $\begin{array}{l}\text { Approx. } \\
\text { Loss } \\
\text { (dB) }\end{array}$ \\
\hline$\overline{\text { Air }}$ & 1 & 0 & 0 & 0 \\
\hline Freshwater & 81 & $10^{-4}$ to $3 \times 10^{-2}$ & 0.018 to 5.39 & 0.037 to 11 \\
\hline Sucwater & 81 & 4 & 719 & 653 \\
\hline Freshwater Ice & 4 & $10^{-5}$ to $10^{-3}$ & 0.0018 to 0.18 & 0.018 to 1.8 \\
\hline Limestone (dry) & 7 & $10^{-9}$ & $1.8 \times 10^{-7}$ & $1.3 \times 10^{-6}$ \\
\hline Granite (dry) & 5 & $10^{-8}$ & $1.8 \times 10^{-6}$ & $1.6 \times 10^{-5}$ \\
\hline Bedded Salt & 3 to 6 & $10^{-5}$ to $10^{-4}$ & $\begin{array}{l}0.0018 \text { to } \\
0.018\end{array}$ & $\begin{array}{l}0.014 \text { to } \\
0.21\end{array}$ \\
\hline Permafrost & 4 to 8 & $10^{-4}$ to $10^{-2}$ & 0.018 to 1.8 & 0.12 to 18 \\
\hline Sand (dry) & 4 to 6 & $10^{-7}$ to $10^{-3}$ & $\begin{array}{l}1.8 \times 10^{-5} \text { to } \\
0.18\end{array}$ & $\begin{array}{l}1.4 \times 10^{-4} \text { to } \\
1.8\end{array}$ \\
\hline Sand (water saturated) & 30 & $10^{-4}$ to $10^{-2}$ & 0.018 to 1.8 & 0.06 to 6 \\
\hline Silt (water saturated) & 10 & $10^{-3}$ to $10^{-2}$ & 0.18 to 1.8 & 1.1 to 11 \\
\hline Rich Agricu'tural Land & 15 & $10^{-2}$ & 1.8 & 8.7 \\
\hline Clay (water saturated) & 8 to 12 & $10^{-2}$ to 1 & 1.8 to 180 & 9.7 to 338 \\
\hline San Antonio Clay Loam (5\% water) & 5.2 & 0.011 & 2 & 17 \\
\hline San Antonio Clay Loam (10\% water) & 14.5 & 0.061 & 11 & 51 \\
\hline San Antonio Clay Loam (20\% water) & 29 & 0.167 & 30 & 93 \\
\hline
\end{tabular}


Some comments are in order.

- For most materials, the dielectric constant $\varepsilon$ (both real and imaginary parts), and hence the refraction index $\eta$, vary fairly slowly with frequency [7][6][3][8][13]. Therefor, the overt contribution of $\omega$ in equations (6) and (7) will probably dominate the frequency dependence of loss. That is, losses will generally be more at higher frequencies, and less at lower frequencies. To a first order, for frequencies near $100 \mathrm{MHz}$, the losses in $\mathrm{dB}$ in table 1 will be scaled linearly with $\omega$, according to equation (7). The result is generally that wideband signals will have their low frequencies emphasized, and higher frequencies tapered. This has an effect analogous to lowering the center frequency, and constricting the bandwidth of the radar echo.

- The relative permeability, often presumed to be unity, can also be significant for some soils. Certainly, in some parts of the U.S. desert southwest, the soils seem to exhibit nontrivial magnetic properties [11].

- The variability of table 1's materials' properties is fairly wide ranging. Since a radar imaging system depends on these par ameters to maintain coherence, this would suggest that a radar imaging system may need to be 'tuned' to the material within which is to be imaged. That is, a priori knowledge of soil parameters is certainly desired, and for some soils may even be required, in order to select appropriate radar parameters and form a reasonable image.

- It is not adequate to know only that a soil is 'clay', 'silt', or 'sand', because dielectric parameters will vary widely within these classes.

- Some measurement techn:ques for relative dielectric constant $\varepsilon$ just automatically presume $\mu=1$, without actually independently measuring $\mu$. These techniques might only measure impedance, only measure the refraction index, only measure loss, etc. A nonunity $\mu$ then has the effect of scaling (perhaps with a complex number) the actual relative complex dielectric constant $\varepsilon$. Measured dielectric values should come with a pedigree as to how they were measured, or at least account for the magnetic permeability. 


\section{AN AIRBORNE SYNTHETIC APERTURE RADAR PHASE HISTORY MODEL}

In considering synthetic apertures, and target locations not directly beneath the CRP, figure 1 needs to be modified and some new definitions made. We will now adopt the geometry of figure 8 .

The parameters of figure 8 are defined as follows.

The CRP is the central reference point, and defines the origin for this geometry.

The dielectric boundary is the $x-y$ plane, with free space above it.

The radar is located in free space with vector $\boldsymbol{r}_{c}$, and has cartesian coordinates $\left(\left\|r_{c}\right\| \cos \psi_{c} \sin \alpha,-\left\|r_{c}\right\| \cos \psi_{c} \cos \alpha,\left\|r_{c}\right\| \sin \psi_{c}\right)$.

The target is $s_{d}$ meters into the lossy half space, and located at cartesian coordinates $\left(s_{x}, s_{y},-s_{d}\right)$. The vector $s$ identifies the surface projection of the target, and points to cartesian coordinates $\left(s_{x}, s_{y}, 0\right)$.

The vector $\boldsymbol{r}_{\boldsymbol{s}}$ is the difference between $\boldsymbol{r}_{\boldsymbol{c}}$ and $\boldsymbol{s}$, and $\psi_{s}$ is the angle between $\boldsymbol{r}_{\boldsymbol{s}}$ and the $x-y$ plane.

We will assume that the assumptions made for equation (2) still apply over all target positions of interest. Therefor, because the target is no longer beneath the CRP, equation (2) becomes

$$
\tau_{s} \approx \frac{2}{c}\left(\left\|r_{s}\right\|+\operatorname{Real}\left[s_{d} \eta \sqrt{1-\left(\frac{\cos \psi_{s}}{\eta}\right)^{2}}\right]\right) .
$$

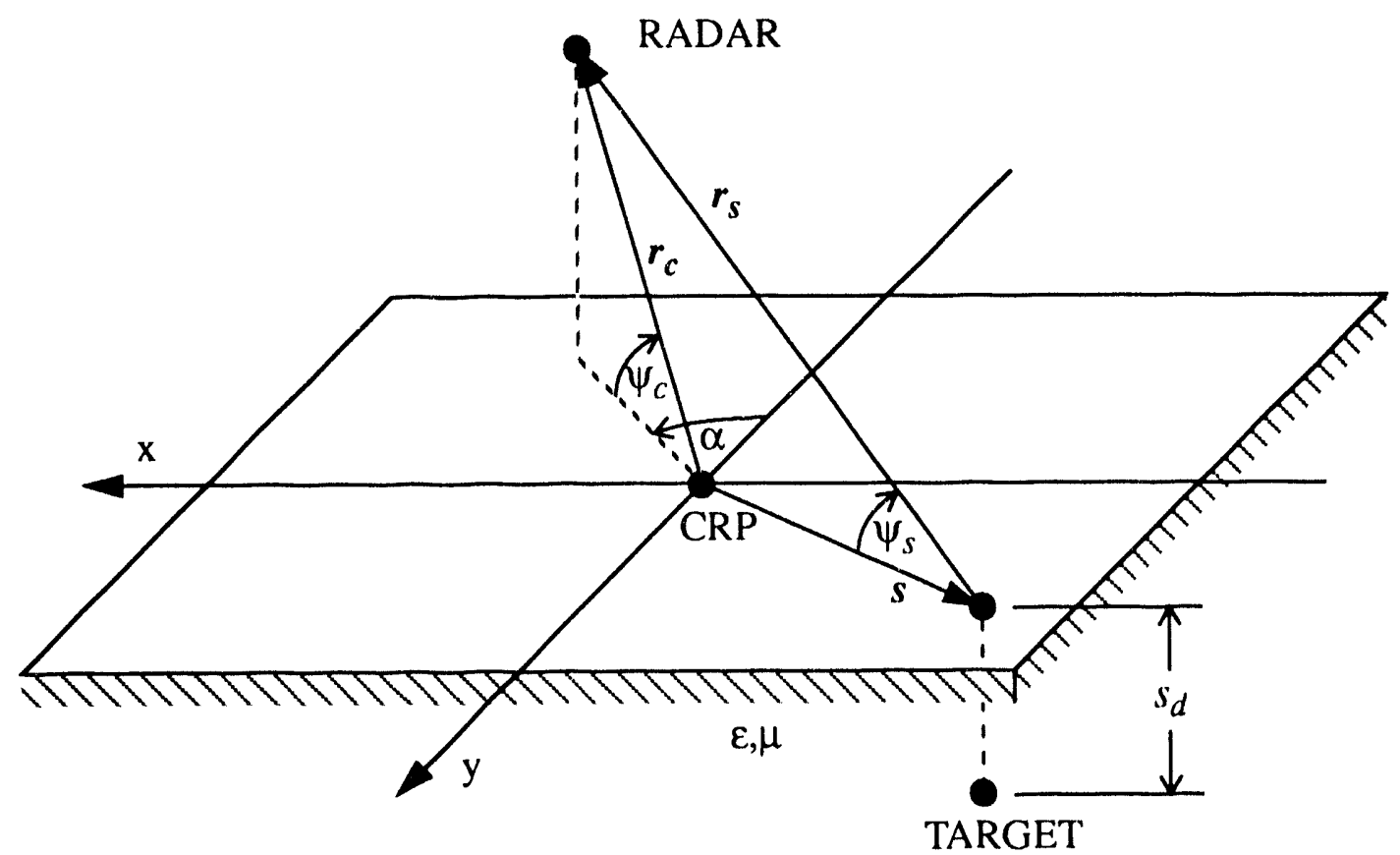

Figure 8. Geometry for SAR phase history model 


\subsection{The Phase History Model}

This development will be for a spotlight mode SAR. Consider a transmitted signal from the radar

$$
X_{T}=G(t) \cos \left(\omega t+\frac{\gamma}{2} t^{2}\right)
$$

where $G(t)=$ transmitted pulse envelope,

$\omega=$ transmitted center frequency, and

$\gamma=$ transmitted chirp rate.

The received echo from the target will then be

$$
X_{R}=A\left(r_{s}, s_{d}\right) G\left(t-\tau_{s}\right) \cos \left(\omega\left(t-\tau_{s}\right)+\frac{\gamma}{2}\left(t-\tau_{s}\right)^{2}\right),
$$

where $A\left(r_{s}, s_{z}\right)=$ the cumulative two-way losses in signal strength.

At this point we are more interested in the character of the signal, and so will ignore dispersion and its mitigation for the moment, assuming it to be inconsequential.

Demodulating and deramping this signal into a video signal with $\mathrm{I}$ and $\mathrm{Q}$ components yields

$$
X_{V}=A\left(r_{s}, s_{d}\right) G\left(t-\tau_{s}\right) \exp \left\{j\left(\left[\omega+\gamma\left(t-\tau_{m}\right)\right]\left(\tau_{m}-\tau_{s}\right)+\frac{\gamma}{2}\left(\tau_{m}-\tau_{s}\right)^{2}\right)\right\} \text {. }
$$

The parameter $\tau_{m}$ is a time offset for the local oscillator signal, which is explicitly

$$
X_{L O}=\exp \left\{-j\left(\omega\left(t-\tau_{m}\right)+\frac{\gamma}{2}\left(t-\tau_{m}\right)^{2}\right)\right\} .
$$

We will select $\tau_{m}$ as follows. Let

$$
\tau_{m}=\frac{2}{c}\left(\left\|r_{\mathrm{c}}\right\|+\operatorname{Real}\left[d \hat{\eta} \sqrt{1-\left(\frac{\cos \psi_{c}}{\hat{\eta}}\right)^{2}}\right]\right)
$$

where $\quad \hat{\eta}=$ estimate of complex refraction index $\eta$, and

$$
d=\text { depth at which we will focus the SAR image. }
$$

The second phase term in equation (11) is the residual phase error term, and in many cases may be ignored. We will do so here. 
Plugging equations (8) and (13) into (11) then yields

$$
\begin{gathered}
X_{V}=A\left(\boldsymbol{r}_{s}, s_{d}\right) G\left(t-\tau_{s}\right) \exp \left\{j \frac{2}{c}\right. \\
\left.\left(\omega+\gamma\left(t-\tau_{m}\right)\right)\left(\left\|r_{\mathrm{c}}\right\|-\left\|r_{s}\right\|+\operatorname{Real}\left[d \hat{\eta} \sqrt{1-\left(\frac{\cos \psi_{c}}{\hat{\eta}}\right)^{2}}\right]-\operatorname{Real}\left[s_{d} \eta \sqrt{1-\left(\frac{\cos \psi_{s}}{\eta}\right)^{2}}\right]\right)\right\} .
\end{gathered}
$$

Making the typical polar-format assumptions for $\left\|r_{\mathrm{c}}\right\|-\left\|r_{\mathrm{s}}\right\|$, that the target area is small enough so $\psi_{s} \approx \psi_{c}$, and our dielectric estimate is good enough so $\hat{\eta} \approx \eta$, we can then simplify a little further, and

$$
\begin{aligned}
& X_{V}=A\left(\boldsymbol{r}_{s}, s_{d}\right) G\left(t-\tau_{s}\right) \exp \left\{j \frac{2}{c}\right. \\
& \left.\left(\omega+\gamma\left(t-\tau_{m}\right)\right)\left(\cos \psi_{c}\left(s_{x} \sin \alpha-s_{y} \cos \alpha\right)+\left(d-s_{d}\right) \hat{\eta}_{R}\left(1-\frac{\cos ^{2} \psi_{c}}{2\|\hat{\eta}\|^{2}}\right)\right)\right\} .
\end{aligned}
$$

Now, to examine the effects of 2-dimensional synthetic aperture, consider small perturbations of $\alpha$ evenly about zero, and small perturbations of $\psi_{c}$ evenly about $\psi_{c 0}$, that is

$$
\cos \psi_{c}=\cos \left(\psi_{c 0}-\Delta \psi\right) \approx \cos \psi_{c 0}+\left(\sin \psi_{c 0}\right) \Delta \psi
$$

and, for the moment, making the typical linear range-doppler assumption

$$
\left(s_{x} \sin \alpha-s_{y} \cos \alpha\right) \approx s_{x} \Delta \alpha-s_{y} .
$$

Defining $\gamma\left(t-\tau_{m}\right) \equiv \Delta \omega$, and putting (16) and (17) into equation (15) yields

$$
\begin{aligned}
& X_{V}=A\left(\boldsymbol{r}_{s}, s_{d}\right) G\left(t-\tau_{s}\right) \exp \left\{j \frac{2}{c}\right. \\
& \left.(\omega+\Delta \omega)\left(\left(\left(\sin \Psi_{c 0}\right) \Delta \psi+\cos \psi_{c 0}\right)\left(s_{x} \Delta \alpha-s_{y}\right)+\left(d-s_{d}\right) \hat{\eta}_{R}\left(1-\frac{\cos ^{2} \Psi_{c 0}}{2\|\hat{\eta}\|^{2}}\right)\right)\right\} .
\end{aligned}
$$

We define $\rho_{x}, \rho_{y}$, and $\rho_{d}$ to be the resolutions at the focal point $(0,0,-d)$ in the $s_{x}, s_{y}$, and $s_{d}$ directions respectively. Then the resolutions are approximately given by

$$
\begin{aligned}
& \rho_{x} \approx \frac{\lambda}{2 \cos \psi_{c 0}\left(2 \Delta \alpha_{\max }\right)}, \\
& \rho_{y} \approx \frac{1}{\frac{2}{\lambda} \sin \psi_{c 0}\left(2 \Delta \psi_{\max }\right)+\frac{2 B}{c} \cos \psi_{c 0}}
\end{aligned}
$$

and 
$\rho_{d} \approx \frac{2}{2 B \eta_{R}\left(1-\frac{\cos ^{2} \psi_{c 0}}{2\|\eta\|^{2}}\right)}$

where

$B=\frac{2 \Delta \omega_{\max }}{2 \pi}=$ bandwidth of transmitted signal in $\mathrm{Hz}$,

$\lambda=\frac{2 \pi c}{\omega}=$ nominal wavelength of transmitted signal,

$\left(2 \Delta \psi_{\max }\right)=$ total elevation aperture angle, and

$\left(2 \Delta \alpha_{\max }\right)=$ total azimuth aperture angle.

At this point we observe the following.

- We recognize that if the target were at the surface $\left(s_{z}=0\right)$ and our focal point were at the surface $(d=0)$, then equation (15) would reduce to the common polar-format representation, and equation (18) would reduce to the common linear range-doppler model.

- From the model of equation (18), apertures in $\Delta \alpha$ will resolve and place $s_{x}$, apertures in $\Delta \psi$ will resolve and place $s_{y}$ and apertures in $\Delta \omega$ will resolve and place $s_{d}$. If the presumption is made that $s_{d}=d$, such as with surface imaging SARs, then an aperture in $\Delta \omega$ will resolve and accurately place $s_{y}$ In such a case $d \neq s_{d}$ manifests itself as an offset in the placement of $s_{y}$ and possibly as a misfocus as well (the well known layover effect). Without an aperture in $\Delta \psi, s_{y}$ and $s_{d}$ are not separable. However, given that $s_{d}=d$, apertures in $\Delta \psi$ and $\Delta \omega$ could be combined in such a way as to improve resolution and placement accuracy in $s_{y}$, beyond the limits imposed by bandwidth or elevation aperture each alone [12]. That is, if $s_{d}=d$, then sampling at different frequencies is equivalent to sampling at different depression angles, as far as resolving and placing $s_{y}$ is concerned.

- The refraction index $\eta$ scales only the $s_{d}$ component of the target position, and not $s_{x}$ nor $s_{y}$. From (19) and (20) it is clear that resolution in the horizontal ( $x$ and $y$ ) directions is unaffected by dielectric properties within the lossy half space. However, (21) shows that depth resolution will be scaled by approximately $1 / \eta_{R}$. Buried targets will appear bigger in the depth direction only, not in the lateral $(\mathrm{x}, \mathrm{y})$ directions. The resolution enhancement in the depth direction may be some consolation for any misfocus caused by dispersion in the dielectric.

- By the same argument used for resolution, the patch size in the $x$ and $y$ directions is largely unaffected by target depth or dielectric properties.

- Even with $\Delta \omega=0$, we are fully capable of locating $s_{x}$ and $s_{y}$ with high precision and resolution. This mode is termed 'holographic SAR' [9]. If the 2-D aperture is an arc, then this is called 'Narrow Band SAR' [10]. 
- Equations (14), (15), and (18) are all just different models with different degrees of approximation to (11). The appropriateness of any given model depends on the validity of the assumptions made in deriving it. These, and perhaps other models, are all usable each in their own circumstance.

\subsection{Impulse Response in 3 Dimensions}

Traditional Synthetic Aperture Radar maps the radar reflectivity of a 2-dimensional surface, and therefor is typically only interested in impulse responses and their sidelobe structure on that surface. Apart from displacements due to layover effects of objects not on the desired surface, 3dimensional impulse responses are generally of little concern. However, the ground, that we have modelled as a lossy half space, is inherently a 3 -dimensional image space, where a target's depth is rarely presumed or known with an acceptable degree of accuracy, and an estimate of which should be apparent from the visualization that is the image. Therefor an understanding of a target's 3-dimensional impulse response (3D-IPR) is essential in evaluating any data collection geometry, processing algorithm, or imaging scheme.

\subsection{Some Collection Geometry Examples}

In this section we generate some phase histories for various data collection geometries using equations (8), (11), and (13). Then, given these phase histories, we form an image using a model that approximates (11). We shall consider a single point target scatterer, and ignore any surface scattering from the dielectric boundary, so that we may illustrate the 3D-IPR of a buried target by itself. The model we shall use also ignores losses, and is

$$
\begin{aligned}
& X_{V}=A\left(r_{s}, 0\right) G\left(t-\tau_{s}\right) \exp \left\{j \frac{2}{c}\right. \\
& \left.(\omega+\Delta \omega)\left(\cos \left(\psi_{c 0}-\Delta \psi\right)\left(s_{x} \sin \Delta \alpha-s_{y} \cos \Delta \alpha\right)+\left(d-s_{d}\right) \hat{\eta}_{R}\right)\right\}
\end{aligned}
$$

The rational for using this model is that it lends itself readily to a 3-dimensional version of polarformat processing, where all the data collected is interpolated to a grid such that the new interpolated data is of the form

$$
X_{V}(k, m, n)=A\left(r_{s}, s_{d}\right) \exp \left\{j \frac{2}{c}\left(s_{x} f_{x} k-s_{\nu} f_{y} m+\left(d-s_{d}\right) f_{d} n\right)\right\},
$$

which can readily be processed into an image using a 3 -dimensional FFT, or equivalent.

The methodology for forming the impulse responses in the following examples is to divide equation (11) by equation (22), and accumulate the result over all phase history samples and frequencies. In essence, we will use (22) as a filter for the data generated with (11). This is done for each pixel location in the resulting image. While this is not how one would ultimately process an image (at least efficiently), it never-the-less illustrates the information content for various data collection geometries, which is what this section is all about. 


\subsubsection{Traditional SAR at UHF}

Consider a traditional SAR with a 1-Dimensional linear spacial aperture, and a linear FM chirp for the frequency aperture. Furthermore, the SAR has the following parameters.

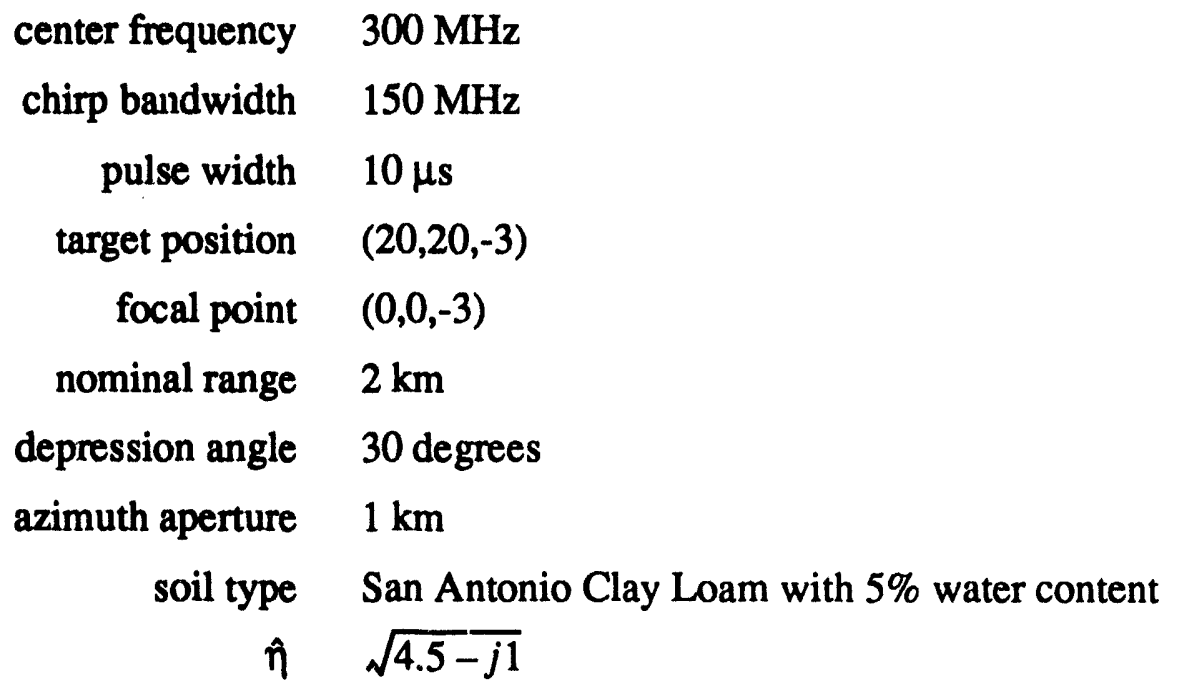

soil type San Antonio Clay Loam with 5\% water content

$\hat{\eta} \quad \sqrt{4.5-j 1}$

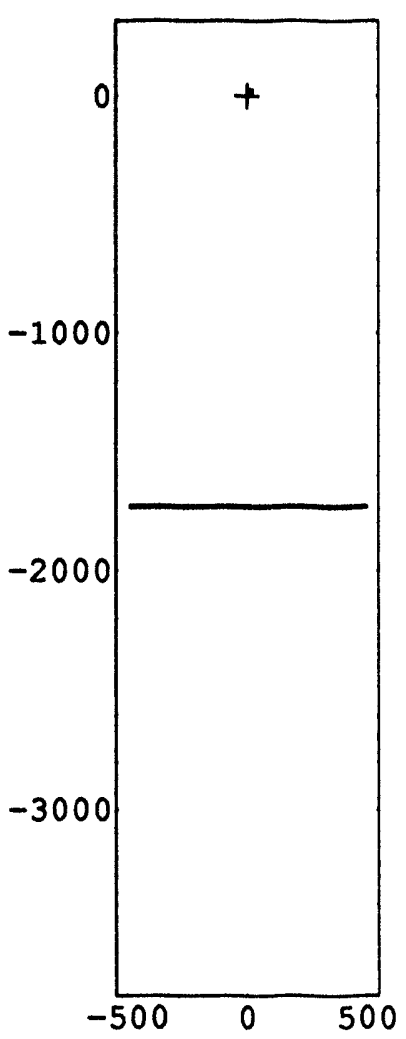

$\mathrm{X}-\mathrm{Y}$ plot of aperture and target area.

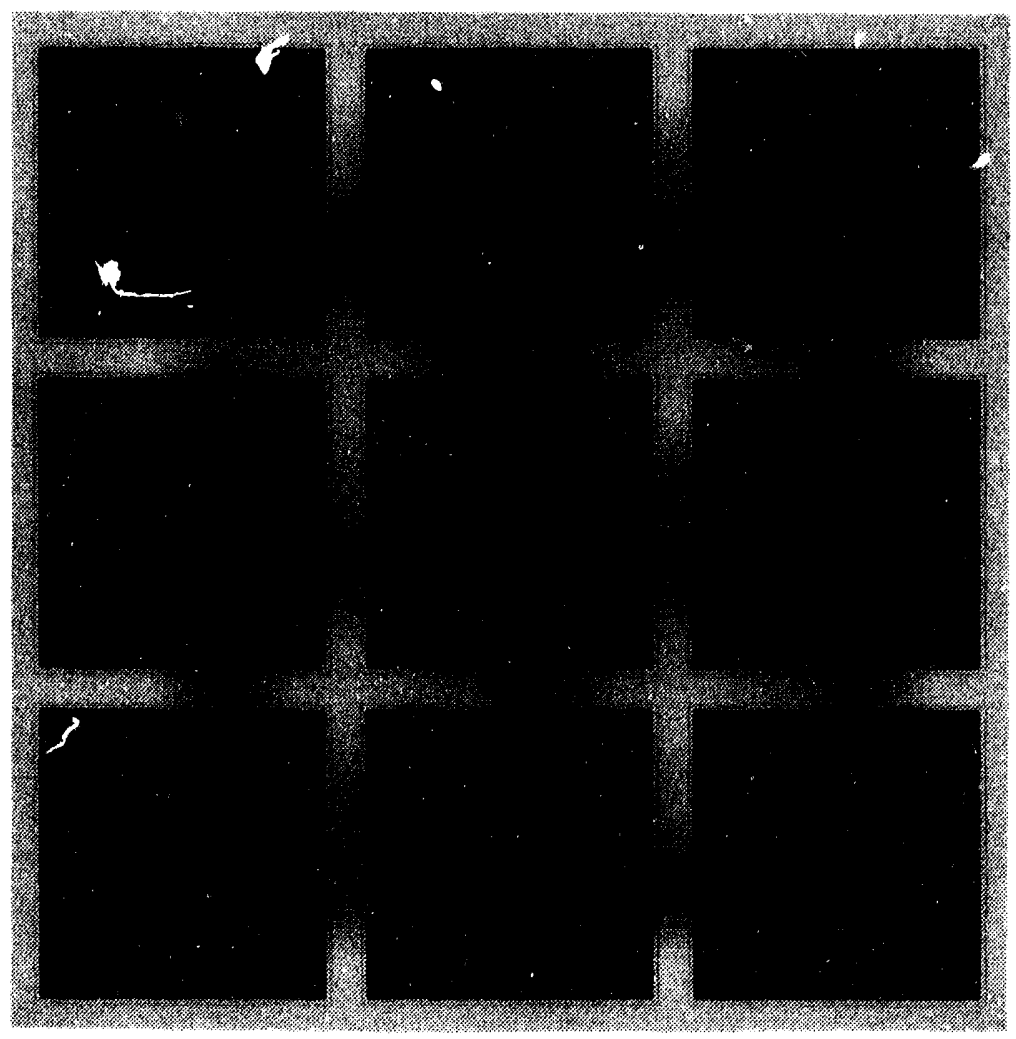

Depth slices at 1, 1.5, 2, 2.5, 3, 3.5, 4, 4.5, and 5 meters. Pixel spacing is $1 \mathrm{~m}$. Image centered on target.

Figure 9. Aperture and Impulse Response for Traditional SAR at UHF. 


\subsubsection{SAR at UHF with Multiple Apertures}

Consider a SAR with multiple 1-Dimensional linear spacial apertures at different orientations, and a linear FM chirp for the frequency aperture, with the following parameters.

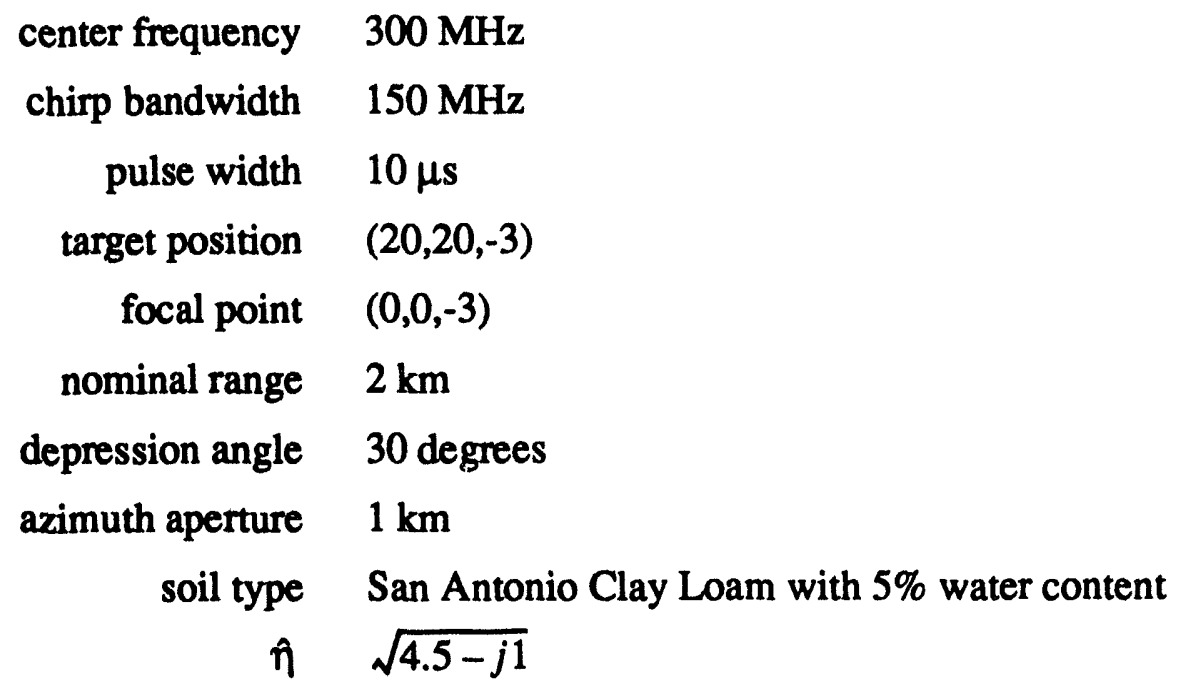

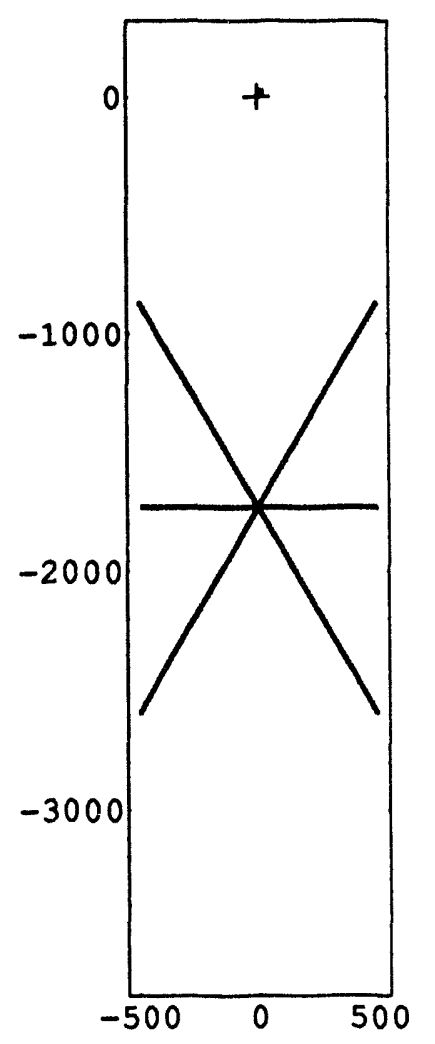

$\mathrm{X}-\mathrm{Y}$ plot of aperture and target area.

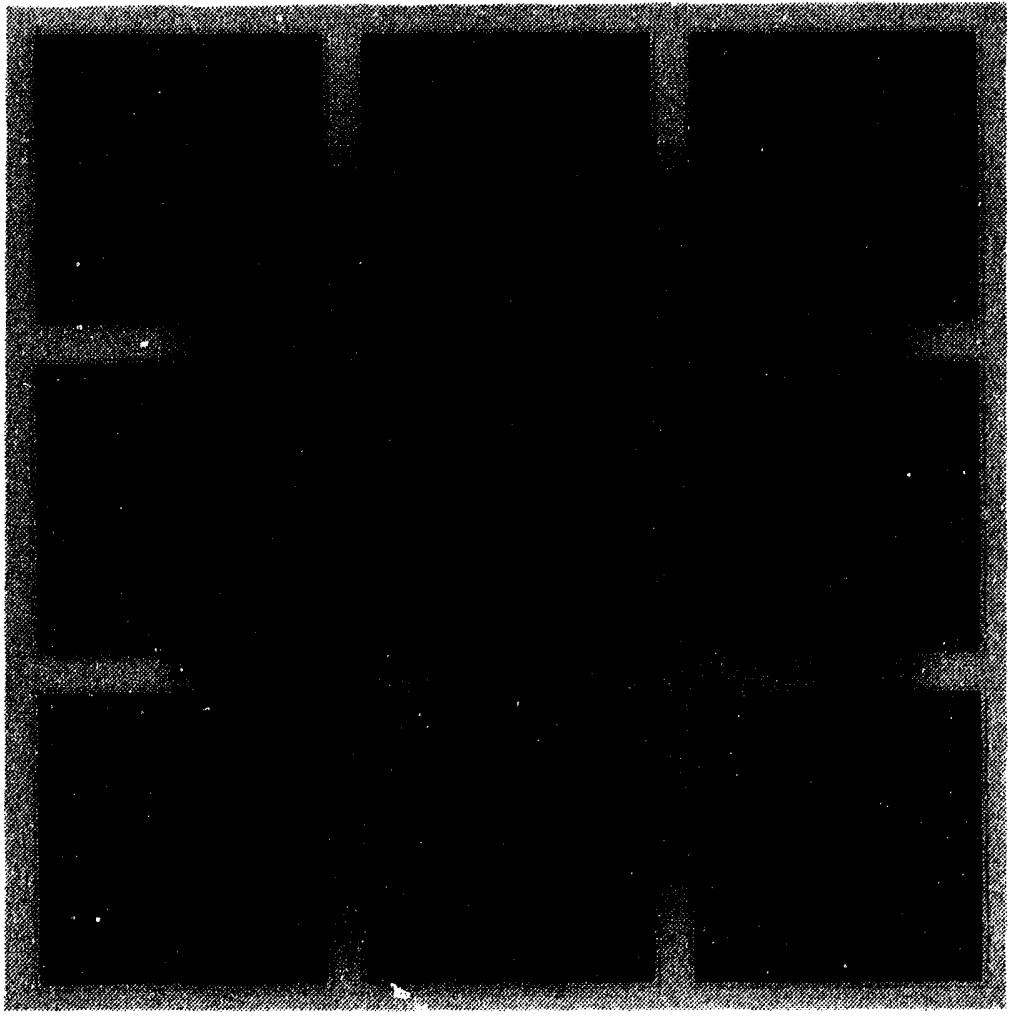

Depth slices at $1,1.5,2,2.5,3,3.5,4,4.5$, and 5 meters. Pixel spacing is $1 \mathrm{~m}$. Image centered on target.

Figure 10. Aperture and Impulse Response for SAR at UHF with multiple apertures. 


\subsubsection{Downward Looking SAR at UHF with Multiple Apertures}

Consider a SAR such as the previous example, but looking downwards at the target area.

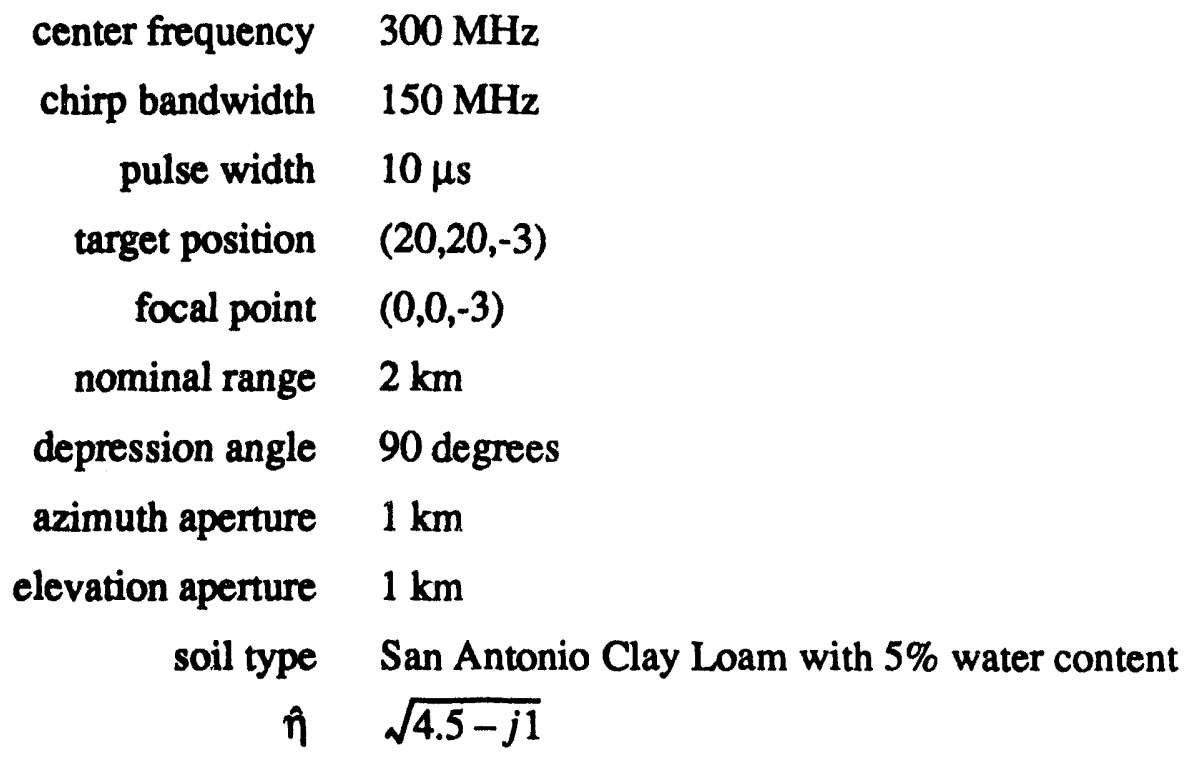

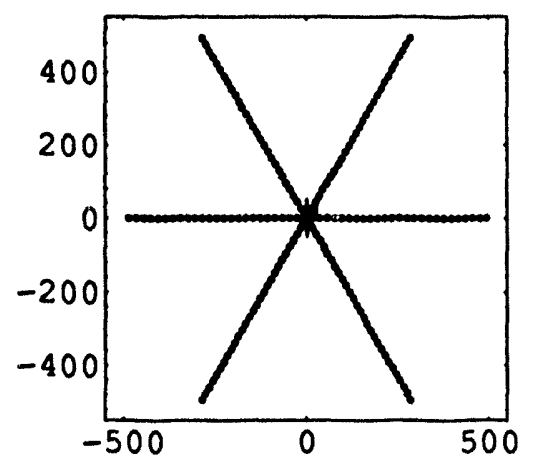

$X-Y$ plot of aperture and target area.

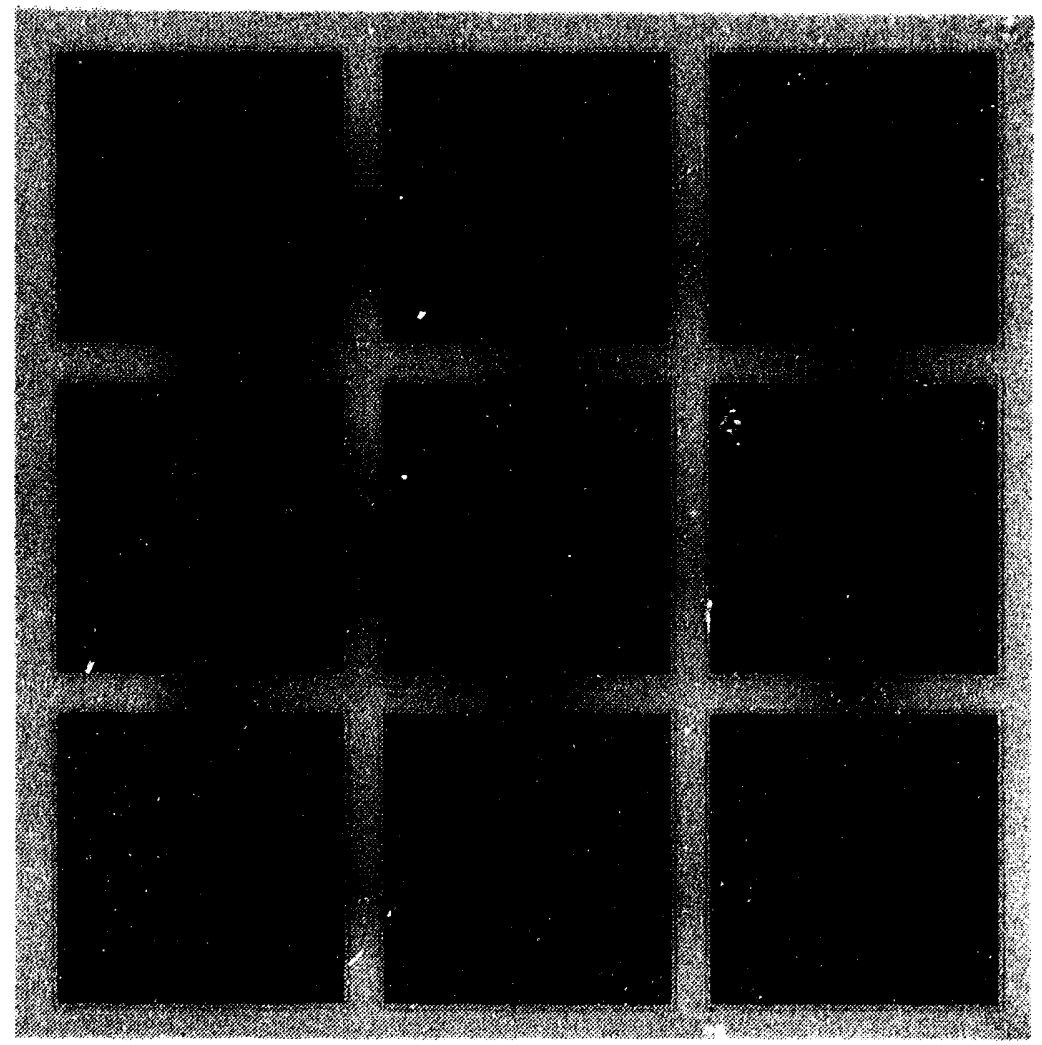

Depth slices at $1,1.5,2,2.5,3,3.5,4,4.5$, and 5 meters. Pixel spacing is $1 \mathrm{~m}$. Image centered on target.

Figure 11. Aperture and Impulse Response for Downward Looking SAR at UHF. 


\subsubsection{Narrow Band Downward Looking SAR at UHF}

Consider a SAR such as the previous example, but looking downwards at the target area, and with zero chirp bandwidth.

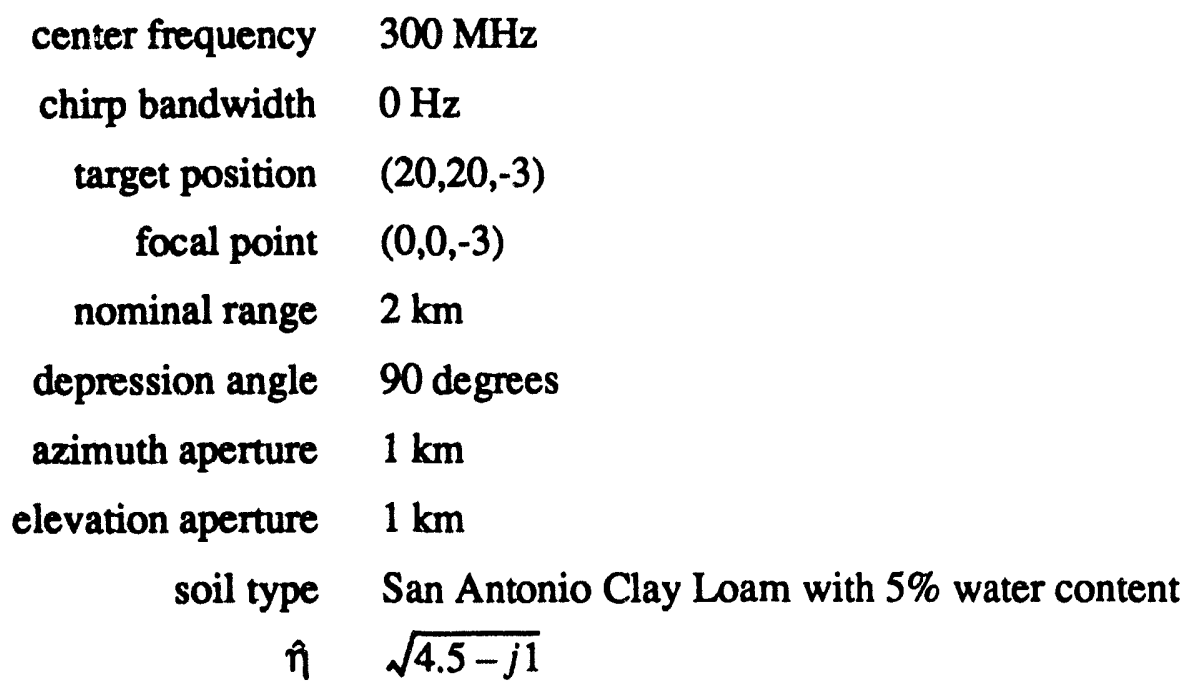

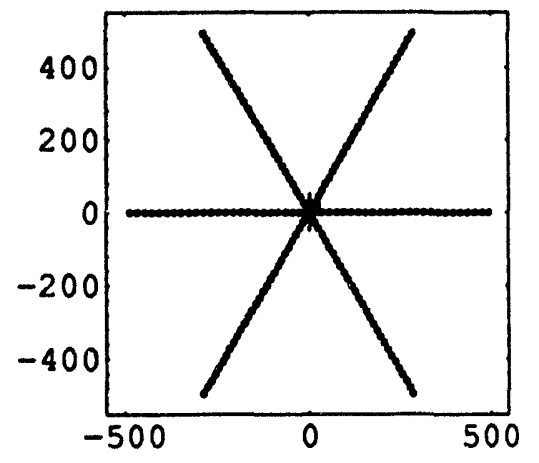

X-Y plot of aperture and target area.

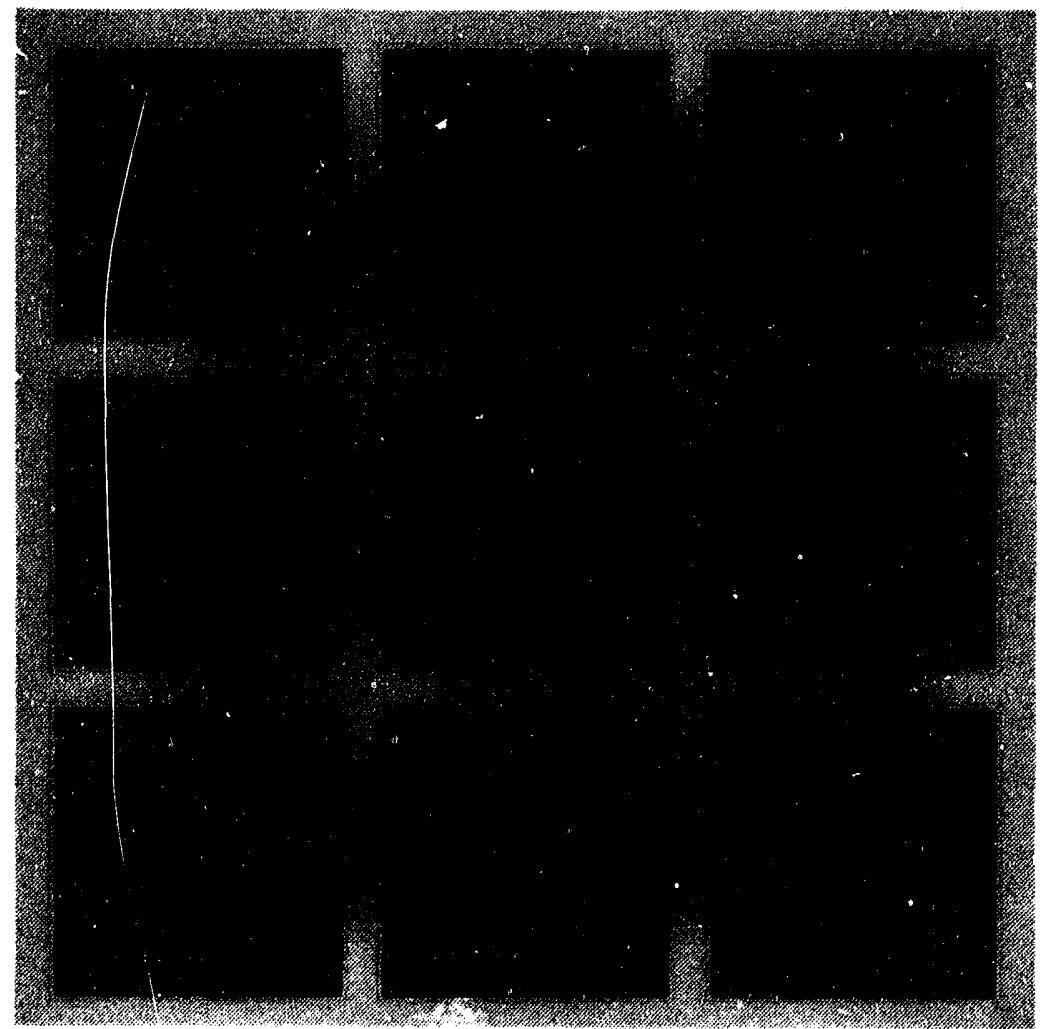

Depth slices at $1,1.5,2,2.5,3,3.5,4,4.5$, and 5 meters. Pixel spacing is $1 \mathrm{~m}$. Image centered on target.

Figure 12. Aperture and Impulse Response for Narrow Band Downward SAR at UHF. 


\subsubsection{Downward Looking SAR at UHF with Multiple Apertures (Shallow Target)}

Consider a downward looking SAR with a shallower target.

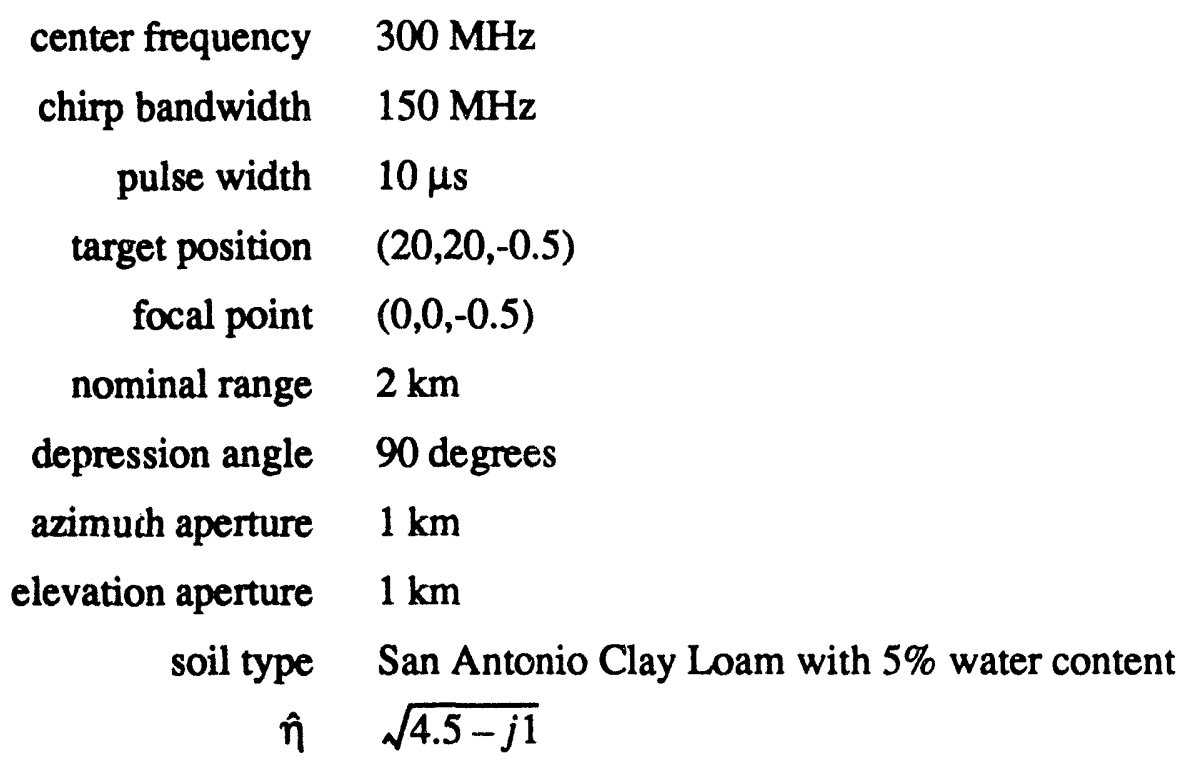

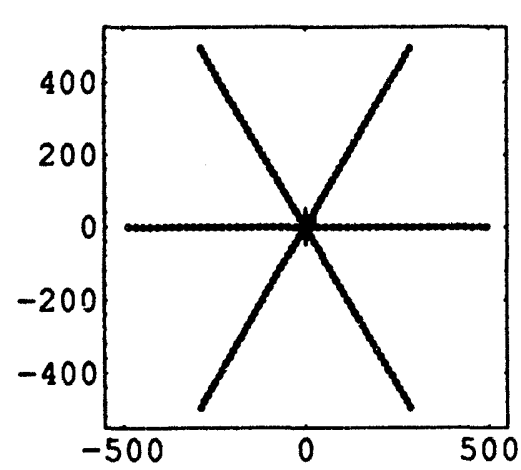

$\mathrm{X}-\mathrm{Y}$ plot of aperture and target area.

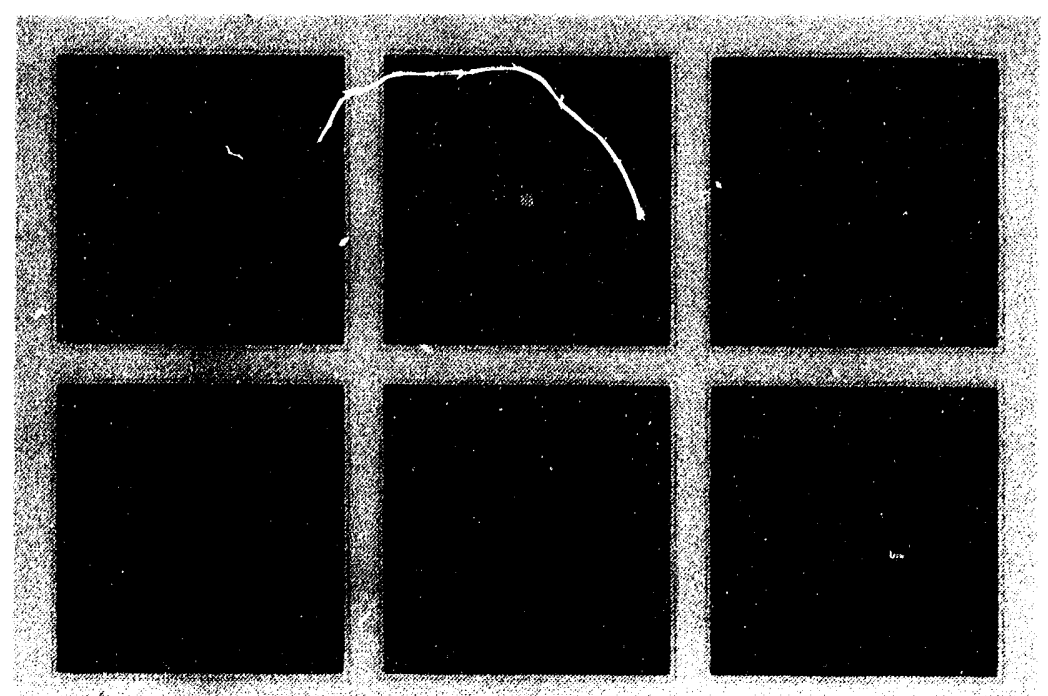

Depth slices at $0,0.5,1,1.5,2$,and 2.5 meters. Pixel spacing is $1 \mathrm{~m}$. Image centered on target.

Figure 13. Aperture and Impulse Response for Downward Looking SAR at UHF. 


\subsubsection{Downward Looking SAR at VHF}

Consider a VHF SAR looking downwards at the target area.

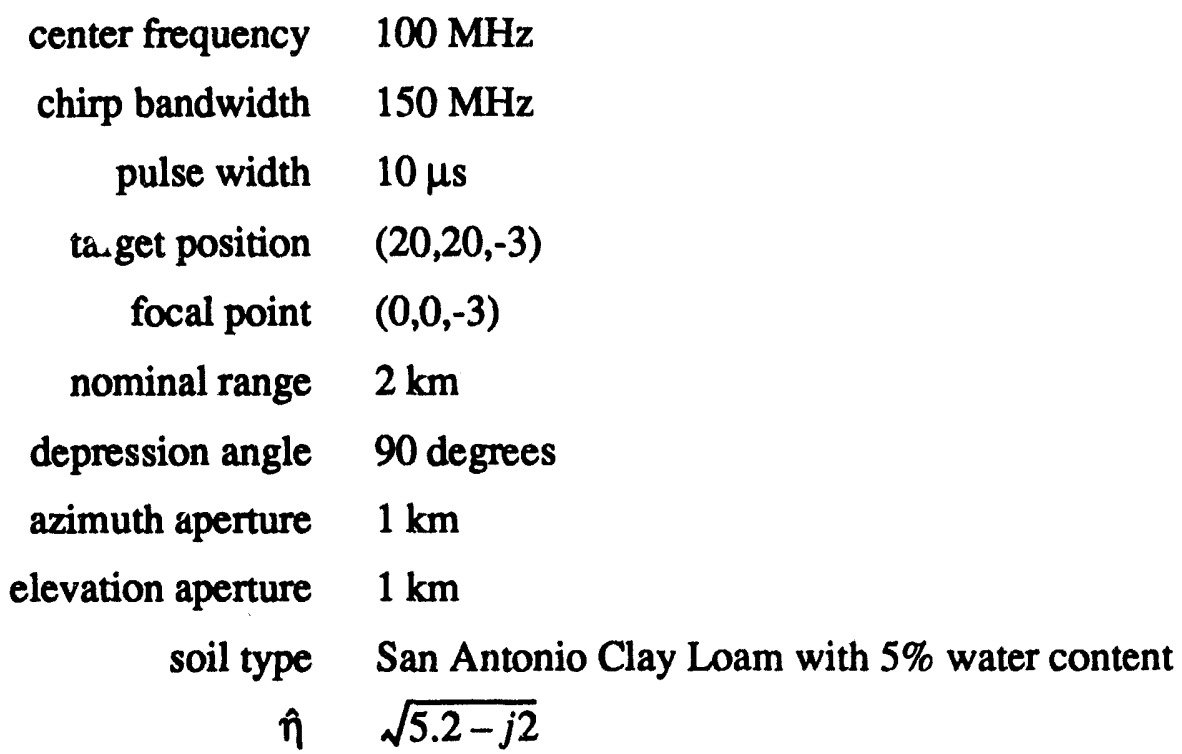

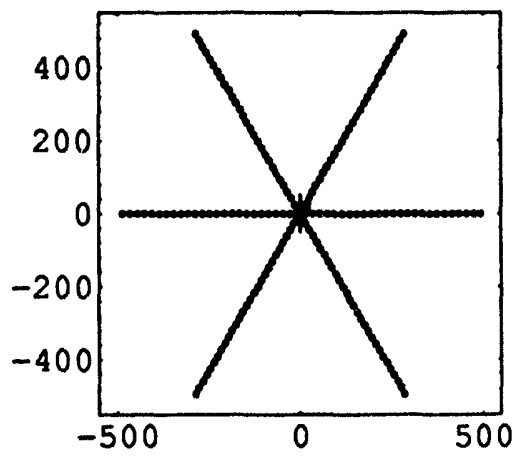

$\mathrm{X}-\mathrm{Y}$ plot of aperture and target area.

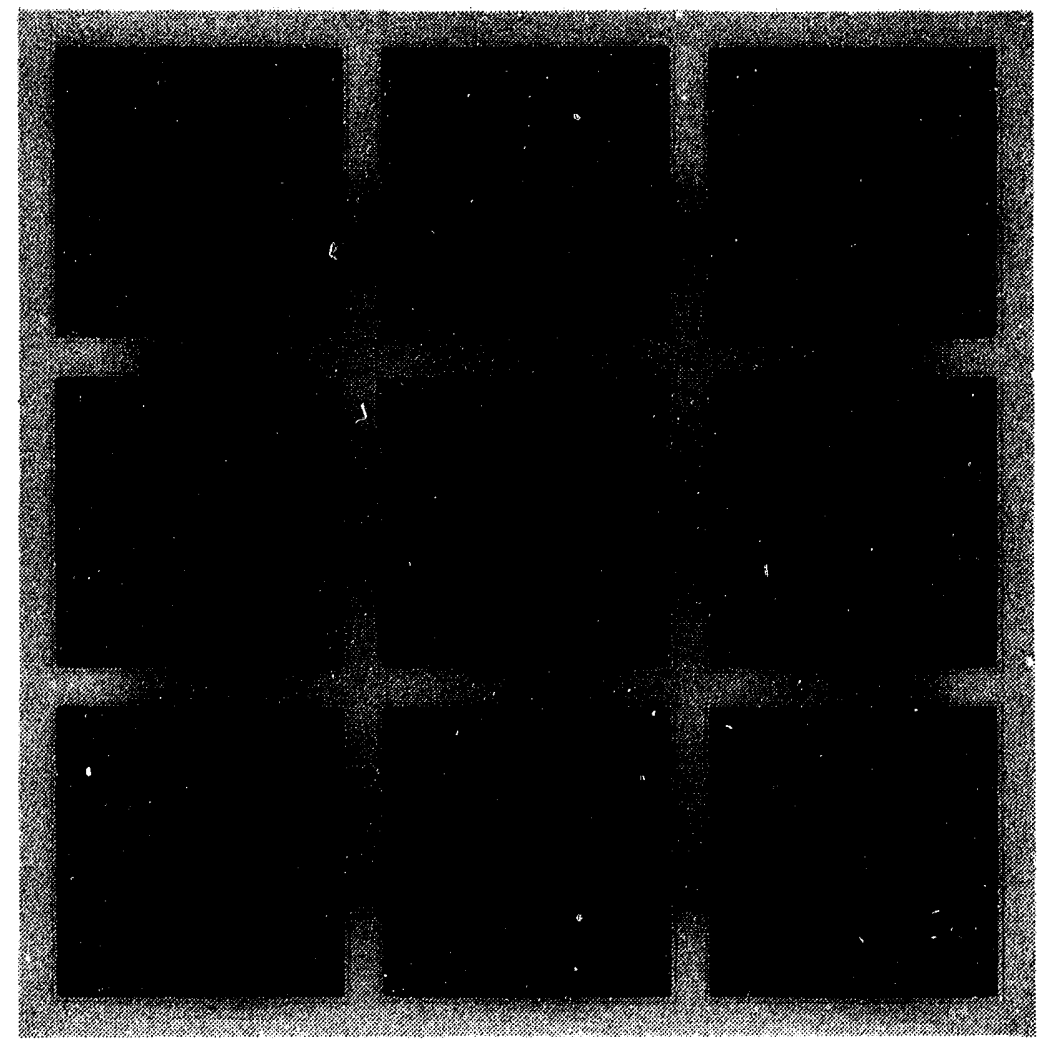

Depth slices at $1,1.5,2,2.5,3,3.5,4,4.5$, and 5 meters. Pixel spacing is $1 \mathrm{~m}$. Image centered on target.

Figure 14. Aperture and Impulse Response for Downward Looking SAR at VHF. 


\subsubsection{Some Comments About the Previous Examples}

- For all examples, the target was presumed to be sufficiently isotropic, and alone. Furthermore, scattering from the dielectric boundary was ignored.

- For all examples, the 3D-IPR was normalized to the brightest pixel over all depth slices.

- L-D vs. 2-D synthetic aperture: Figure 9 shows how a 1-D synthetic azimuth aperture allows ambiguities in $s_{y}$ vs. $s_{d}$, the well known layover effect. Figure 10 shows how this ambiguity may be mitigated with an elevation aperture.

- Sidelobe orientation: Figure 10 shows elevation aperture sidelobes that are tilted due to the 2-D synthetic aperture being offset with a nominal depression angle less than 90 degrees. Figure 11 shows that these sidelobes spread evenly within a depth slice for a vertical aperture.

- Depth resolution: Figure 12 illustrates how a narrow band signal can still resolve the target in two dimensions, but not in all three as in figure 11.

- Dispersion effects: Comparing figures 11 and 13 shows how deeper targets are less resolvable in depth due to dispersion and frequency dependent attenuation effects.

- Center Frequency: Comparing figures 11 and 14 shows how the center operating frequency influences resolutions $\rho_{x}$ and $\rho_{y}$, but not depth resolution $\rho_{d}$.

- With the broad-beamed antennas and large dynamic ranges inherently a part of subsurface imaging radars, the real antennas should be considered effectively isotropic. Therefor, an entire 2-dimensional synthetic aperture would ideally be 'filled in' with a separation of no more than $\lambda / 2$ between spacial samples. This seems impractical for an airborne system trying to cover synthetic apertures of hundreds of meters, or even kilometers in dimension. Therefor sub-sampled arrays need to be considered, perhaps made up of multiple 1-D apertures such as in the examples, or perhaps more complicated patterns such as serpentine or spiral flight paths. The key is that all spacial samples need to be coherent, which has non-trivial ramifications for the accuracy of motion measurement systems. In general, however, the more filled in the 2-D synthetic aperture is, the better control of sidelobes one has. This problem is essentially the same as is encountered with 'thinned arrays' in antenna theory.

- In all cases, the 3D-IPR exhibits 'spilled energy' (sidelobes) in all three dimensions. While always a nuisance, sidelobes in the depth direction are particularly troublesome as they may easily mask a deeper target, since a deeper target's IPR may be significantly attenuated due to propagation loss anyway (that is, attenuated to below the sidelobe level of a shallower target). The traditional means of sidelobe reduction is by using window functions during image formation, but for a lossy medium these window functions would ideally account for the orientation of the sidelobes as well as the lossiness of the medium. 


\section{DYNAMIC RANGE ISSUES IN SIGNAL PROCESSING}

As was seen in the last section, finite apertures, whether spatial or frequency, will yield sidelobes to an impulse response. System non-linearities will also affect sidelobe shapes and levels. These sidelobes may extend to deeper depths, where they may mask the return of a deeper target, which is undesirable. In this section we explore this effect. But first we explore the dynamic range of a Ground Penetrating SAR.

\subsection{Dynamic Range}

Dynamic range is defined as a maximum value divided by a minimum value, and is usually expressed in $\mathrm{dB}$. For a ground penetrating SAR image, the values are radar cross section. The maximum value is the largest radar cross section that still keeps processing components in a sufficiently linear operating region, usually associated with a surface return, and then often defined as a surface clutter return. The minimum value is the smallest radar cross section that still maintains some nominal signal-to-noise ratio (typically $0 \mathrm{~dB}$, or $6 \mathrm{~dB}$ ) in a processed image, usually associated with some target at some depth. The deepest such depth is termed the 'depth horizon'. Dynamic range numbers typically ignore the interaction of target sidelobes. We illustrate dynamic range with the following example.

Example 4. Consider a half space of San Antonio Clay Loam with 5\% water content, with a smooth surface. Furthermore, consider two targets. The first target is a surface reflector with $+10 \mathrm{dBsm}$ cross section. The second target is a $-10 \mathrm{dBsm}$ reflector at a depth of 3 meters. We are using a nominal frequency of $100 \mathrm{MHz}$, and imaging at a nominal depression angle of 60 degrees. From figure 4 , the two way propagation loss is about $48 \mathrm{~dB}$. From reference [2], the two way transmissivity loss is about $2 \mathrm{~dB}$. The required dynamic range for imaging these targets is then the difference in the targets' cross sections plus all the losses (in $\mathrm{dB})$. That is

$$
\text { example dynamic range }=[10-(-10)+48+2]=70 \mathrm{~dB} \text {. }
$$

Clearly, however, if the two targets are vertically aligned with each other (one on top of the other), then the sidelobes from the surface target's response need to be at least $70 \mathrm{~dB}$ down from the mainlobe response at a depth of 3 meters in order to fully use this dynamic range. In such a case, the purity of the signal, system nonlinearities, and the window function that is used in image formation can all severely limit the full use of the dynamic range. For example, if the sidelobes of the surface target are down by only $40 \mathrm{~dB}$, but the deeper target's response is down by $70 \mathrm{~dB}$, then discerning the presence of the deeper target will not be possible. 
Thus far we have been dealing with the dynamic range of the processed image, which includes the noise reduction offered by coherent gain. To calculate the raw dynamic range required of the radar front-end hardware, including the analog-to-digital converters, we need to subtract the coherent processing gain from the processed image dynamic range, and reference the noise floor by adding the minimum required signal-to-noise ratio (SNR) in the final image.

Example 4 (continued). Suppose our SAR offers a total of $60 \mathrm{~dB}$ coherent processing gain, and we wish to maintain a minimum SNR of $6 \mathrm{~dB}$. Then

$$
\text { raw dynamic range }=[70-60+6]=16 \mathrm{~dB} \text {, }
$$

which requires at least 5 bits in the analog-to-digital converter ( 3 bits for signal +1 bit for noise +1 sign-bit), assuming the noise level is set to toggle the least significant bit. Of course, some margin would be prudent.

\subsection{Loss Normalized Depth Impulse Response - Matched Filter Processing}

Equation (6) expresses the power attenuation of a radar echo from a buried target. If we now consider two identical targets buried with a depth difis ience of $\Delta d$, then a resulting 3-D image will show the deeper target with an intensity diminished from the shallower target by

$$
\Delta \approx \exp \left(\frac{2 \omega}{c} \operatorname{Imag}\left[(\Delta d) \eta \sqrt{1-\left(\frac{\cos \psi}{\eta}\right)^{2}}\right]\right)
$$

where $\Delta$ is the ratio of the intensities.

A better understanding may be had by seeing how loss affects SAR processing. To this end, consider a target buried at location $\left(0,0,-s_{d}\right)$. Now consider an ideal system modelled by equation (14) with $d=0$,

$$
X_{V}=A\left(r_{s}, s_{d}\right) G\left(t-\tau_{s}\right) \exp \left\{j \frac{2}{c}(\omega+\Delta \omega)\left(-\operatorname{Real}\left[s_{d} \eta \sqrt{1-\left(\frac{\cos \psi_{c}}{\eta}\right)^{2}}\right]\right)\right\},
$$

and recognize from equation (6) that

$$
A\left(r_{s}, s_{d}\right)=A\left(r_{s}, 0\right) \exp \left\{\frac{2}{c}(\omega+\Delta \omega)\left(\operatorname{Imag}\left[s_{d} \eta \sqrt{1-\left(\frac{\cos \psi_{c}}{\eta}\right)^{2}}\right]\right)\right\}
$$


Putting these together yields

$$
\begin{aligned}
& X_{V}=A\left(r_{s}, 0\right) G\left(t-\tau_{s}\right) \exp \{ \\
& \left.\frac{2}{c}(\omega+\Delta \omega) s_{d}\left(\operatorname{Imag}\left[\eta \sqrt{1-\left(\frac{\cos \psi_{c}}{\eta}\right)^{2}}\right]-j \operatorname{Real}\left[\eta \sqrt{1-\left(\frac{\cos \psi_{c}}{\eta}\right)^{2}}\right]\right)\right\}
\end{aligned}
$$

or equivalently

$$
X_{V}=A\left(r_{s}, 0\right) G\left(t-\tau_{s}\right) \exp \left\{-j \frac{2}{c}(\omega+\Delta \omega) s_{d}\left[\eta \sqrt{1-\left(\frac{\cos \psi_{c}}{\eta}\right)^{2}}\right]\right\}
$$

where the phase is now complex.

For an evenly sampled frequency aperture about $\omega$, consider video samples with frequency

$$
\omega+\Delta \omega=\omega_{\min }+k d \omega
$$

where $k=0 . .(K-1)$.

Now consider a matched filter, where equation (28) is correlated against a target's expected response from depth $d$. For simplicity we shall ignore dispersion and presume constant $\eta$. The output of such a filter is then given by

$$
I P R=A\left(r_{s}, 0\right) \frac{\sin \left[\frac{K d \omega}{c}\left(s_{d}-d\right) \eta \sqrt{1-\left(\frac{\cos \psi_{c}}{\eta}\right)^{2}}\right]}{\sin \left[\frac{d \omega}{c}\left(s_{d}-d\right) \eta \sqrt{1-\left(\frac{\cos \psi_{c}}{\eta}\right)^{2}}\right]} \exp \left[-j \frac{2 \omega}{c}\left(s_{d}-d\right) \eta \sqrt{1-\left(\frac{\cos \psi_{c}}{\eta}\right)^{2}}\right]
$$

Note that a complex $\eta$ affects the IPR's envelope even in the exponential term. The loss due to depth is completely incorporated into this impulse response, that is, this impulse response is loss normalized. What may not be immediately clear, but is never-the-less true, is that for a complex $\eta$, as $d$ increases, ultimately the envelope of the IPR will grow and even surpass the magnitude of the main lobe. Furthermore, this will be true regardless of whether or which window function is used, and regardless of the available dynamic range. The implication is that sidelobes of shallow targets will overshadow mainlobes of identical targets that are deeper. To illustrate this, consider the following two examples. 
Example 5. Consider two identical point targets buried in a lossy half space with a dielectric constant representative of dry sand at $300 \mathrm{MHz}$ and other parameters as listed

relative dielectric constant $2.5-\mathrm{j} 0.025$

relative permeability 1

depression angle 30 degrees

center frequency $\quad 300 \mathrm{MHz}$

bandwidth $\quad 150 \mathrm{MHz}$

video samples $(\mathrm{K}) \quad 128$

target \#1 depth $0 \mathrm{~m}$ (just below the surface)

target \#2 depth $2 \mathrm{~m}$

Figure 15 illustrates the matched filter output for these target's responses. The targets are readily distinguishable.

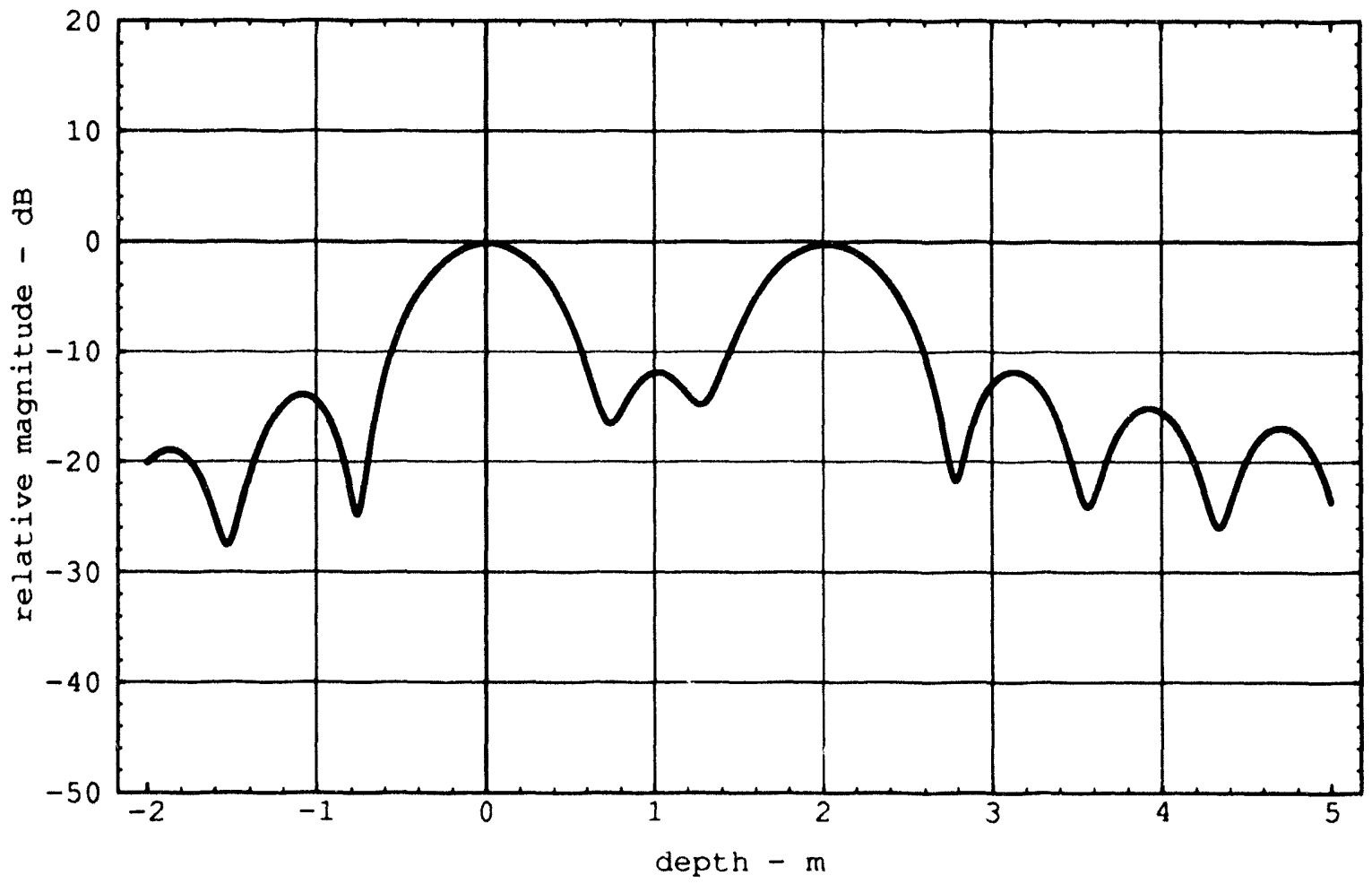

Figure 15. Matched-Filtered IPRs of targets in dry sand. 
Example 6. Now consider the parameters of example 5 except with a dielectric constant representative of San Antonio Clay Loam with 5\% water content at 300 MHz.

relative dielectric constant

$4.5-\mathrm{j} 1$

relative permeability 1

depression angle 30 degrees

center frequency $300 \mathrm{MHz}$

bandwidth $\quad 150 \mathrm{MHz}$

video samples $(\mathrm{K}) \quad 128$

target \#1 depth $0 \mathrm{~m}$ (just below the surface)

target \#2 depth $2 \mathrm{~m}$

Figure 16 illustrates the new matched filter output for these target's responses.

Clearly, the deeper target is masked by the sidelobes of the shallower target. The sidelobes increase as depths get deeper.

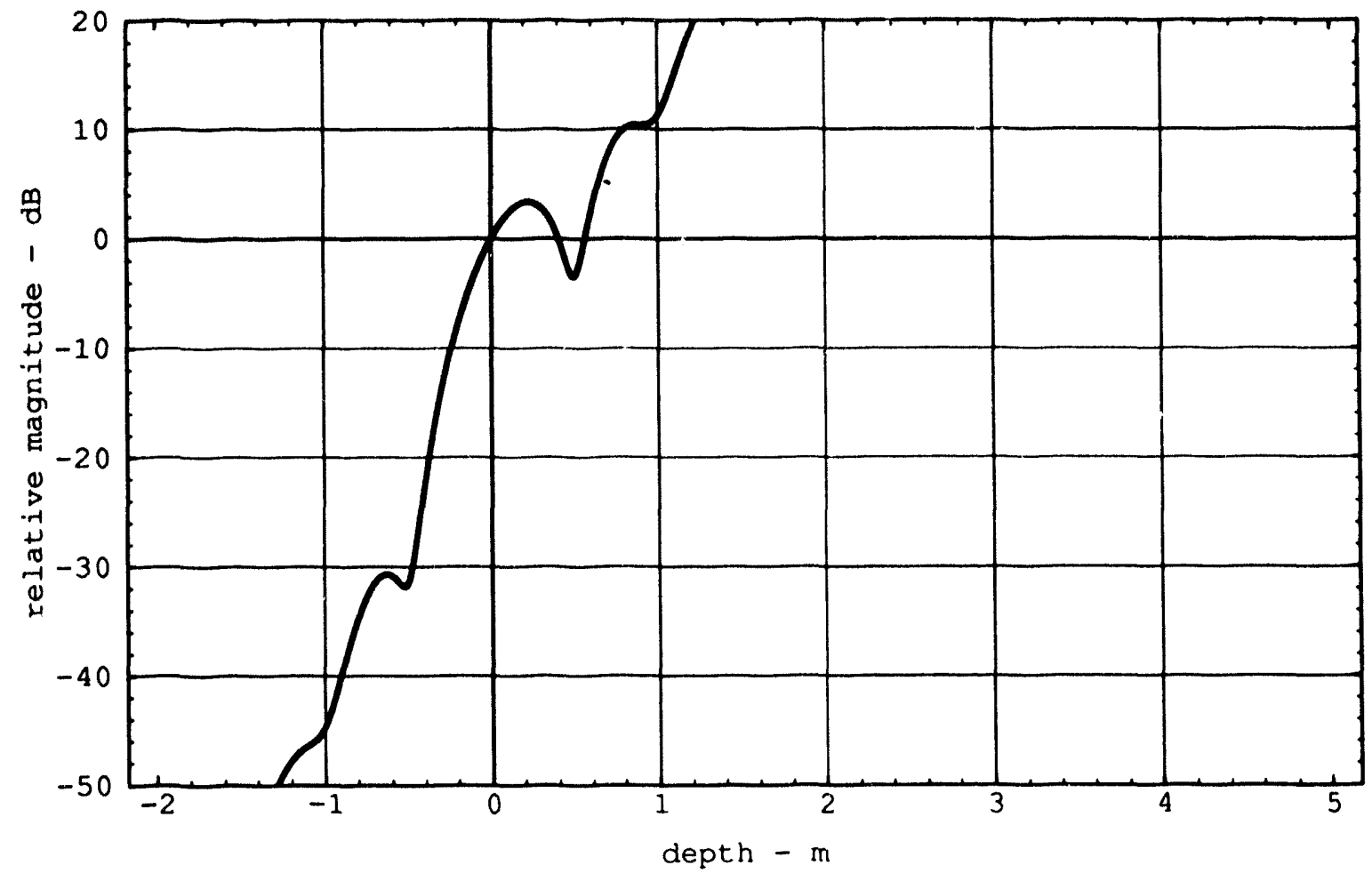

Figure 16. Matched-Filtered IPRs of targets in San Antonio Clay Loam with 5\% water. 


\subsection{Loss Normalized Depth Impulse Response - DFT Processing}

The matched filter of the previous section requires a different filter for every depth of interest. While this approach might be tolerable when only a few depths are of interest, under some conditions other more efficient processing might be applied to resolve depth. To pursue this, consider equation (27) modified by equation (29) and rewritten as

$$
\begin{aligned}
X_{V}=A\left(\boldsymbol{r}_{s}, 0\right) \exp \left\{\frac{2}{c}\left(\omega_{\min }+k d \omega\right) s_{d}\left(\operatorname{Imag}\left[\eta \sqrt{1-\left(\frac{\cos \psi_{c}}{\eta}\right)^{2}}\right]\right)\right\} \\
\cdot \exp \left\{-j \frac{2}{c}\left(\omega_{\min }+k d \omega\right) s_{d}\left(\operatorname{Real}\left[\eta \sqrt{1-\left(\frac{\cos \psi_{c}}{\eta}\right)^{2}}\right]\right)\right\} .
\end{aligned}
$$

The top line of equation (31) concerns amplitude, and the bottom line phase. Clearly, target depth $s_{d}$ is encoded in the frequency (in $k$ ) of line 2 of equation (31). The exponential of line 1 of equation (31) is an amplitude weighting across $k$, much like a window function, but that depends on target depth $s_{d}$. If the soil is not too lossy, the target not too deep, and the fractional bandwidth not too great, then this factor will not vary too much with frequency index $k$, although a depth dependence will remain. That is, equation (31) can be approximated with

$$
\begin{aligned}
& X_{V} \approx A\left(r_{s}, 0\right) \exp \left\{\frac{2 \omega s_{d}}{c}\left(\operatorname{Imag}\left[\eta \sqrt{1-\left(\frac{\cos \psi_{c}}{\eta}\right)^{2}}\right]\right)\right\} \\
& \cdot \exp \left\{-j \frac{2}{c}\left(\omega_{\text {min }}+k d \omega\right) s_{d}\left(\operatorname{Real}\left[\eta \sqrt{1-\left(\frac{\cos \psi_{c}}{\eta}\right)^{2}}\right]\right)\right\} .
\end{aligned}
$$

Resolving $s_{d}$ is then a matter of applying an Inverse Discrete Fourier Transform (IDFT), which acts on line 2 of equation (32). The amplitude dependence on depth $s_{d}$ in line 1 of equation (32) can be compensated thereafter, in what amounts to a loss normalization, or depth calibration step. This can be illustrated with the following examples.

Example 7. Reconsider the targets and parameters of example 5, but now processed with DFT processing. The loss normalized result is displayed in figure 17.

Example 8. Reconsider the targets and parameters of example 6, but using the IDFT after applying a Taylor window, with nbar $=6$ and $-40 \mathrm{~dB}$ sidelobes. The loss normalized result for this soil is displayed in figure 18. 

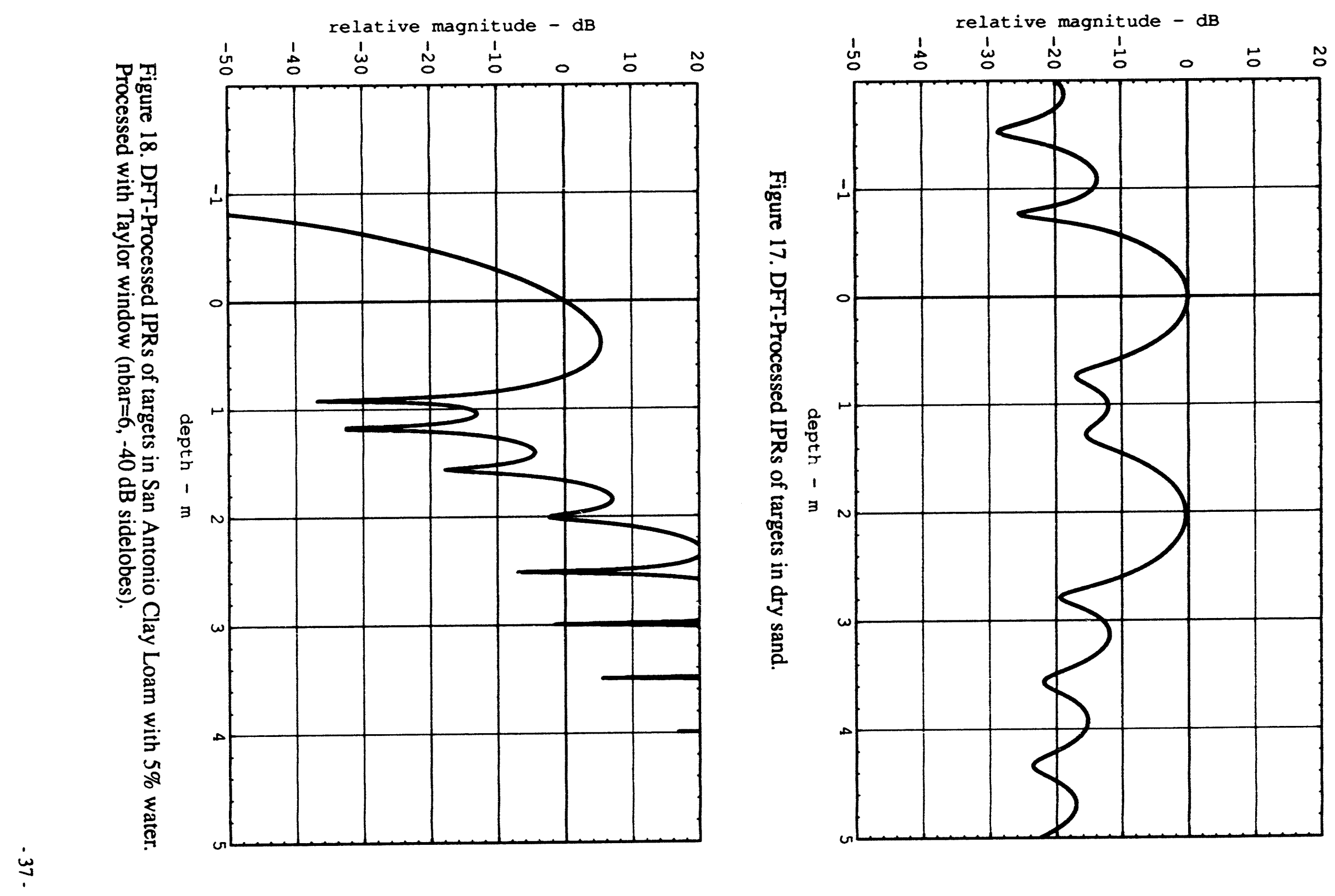
Some observations are in order.

- Between figures 15 and 16 we observe that as a soil becomes lossier, two things happen. The first is that the IPR width as measured at the $-3 \mathrm{~dB}$ points becomes narrower, that is, resolution becomes enhanced. The second is that sidelobes begin to interfere more with the IPR of deeper targets, to the point of overwhelming the deeper target's IPR. This is in spite of dynamic range considerations due to noise.

- Comparing figures 15 and 17 shows that for dry sand, DFT processing is nearly identical to processing with a Matched-Filter. This is true primarily because dry sand is a low loss medium.

- Figure 18 shows that the deeper target is still overwhelmed by the sidelobes of the shallower target even though sidelobes were suppressed by $40 \mathrm{~dB}$. At least another $16 \mathrm{~dB}$ of suppression would be required just to make them comparable with the mainlobe of the deeper target. In fact, for this soil, a window function would have to reduce sidelobes by at least $28 \mathrm{~dB}$ per meter to overcome the effects of loss for any target buried under it. In addition, the mainlobe of the shallow target is broader than that of figure 16 owing to the use of the window function. For traditional window functions, the broadening will generally increase as the sidelobes are further suppressed.

- Sidelobes are a more significant problem for targets buried below a scatterer than for targets buried above a scatterer. But typical window functions are real, and hence yield even IPRs. What is needed is a window function that really suppresses sidelobes on the down side of an IPR, even if at the expense of the sidelobes on the up side of the IPR. Then again, there is no real point to suppressing sidelobes below the depth horizon.

- Sidelobe levels are a function of system non-linearities and the window function used in the processing of the phasy histories into an image. System non-linearities can sometimes be mitigated with auto-focus type algorithms and procedures. Window functions can sometimes be loss compensated, as will be described in the next section. 


\subsection{Loss Compensated Window Functions for Near Sidelobe Suppression}

Consider first some characteristics for the ideal window function. This window function would suppress sidelobes in a loss normalized image to a level significantly below the mainlobe, and it would do so even for surface targets down to at least the depth horizon.

The spectral characteristics of the window itself (prior to loss normalization) can then be inferred, and are illustrated in figure 19. The main features of the window spectrum are identified as follows:

Region 1 are the sidelobes corresponding to sufficiently far above the target that loss normalization will suppress these even further. In this spectrum it is merely important that they be below the mainlobe peak and allow most of the signal energy to remain in the peak, thereby preserving signal-to-noise ratio.

Region 2 contains the area immediately around the mainlobe of the response and sidelobes that correspond to a distance extending downward to the depth horizon. This region will exhibit an exponential taper 'tuned' to the lossiness of the soil.

Region 3 are the sidelobes corresponding to depths below the depth horizon. As with region 2 , in this spectrum it is merely important that they be below the maiplobe peak and thereby allow most of the signal energy to remain in the peak.

Applying a window with this spectrum in DFT-processing (with loss normalization) will ideally

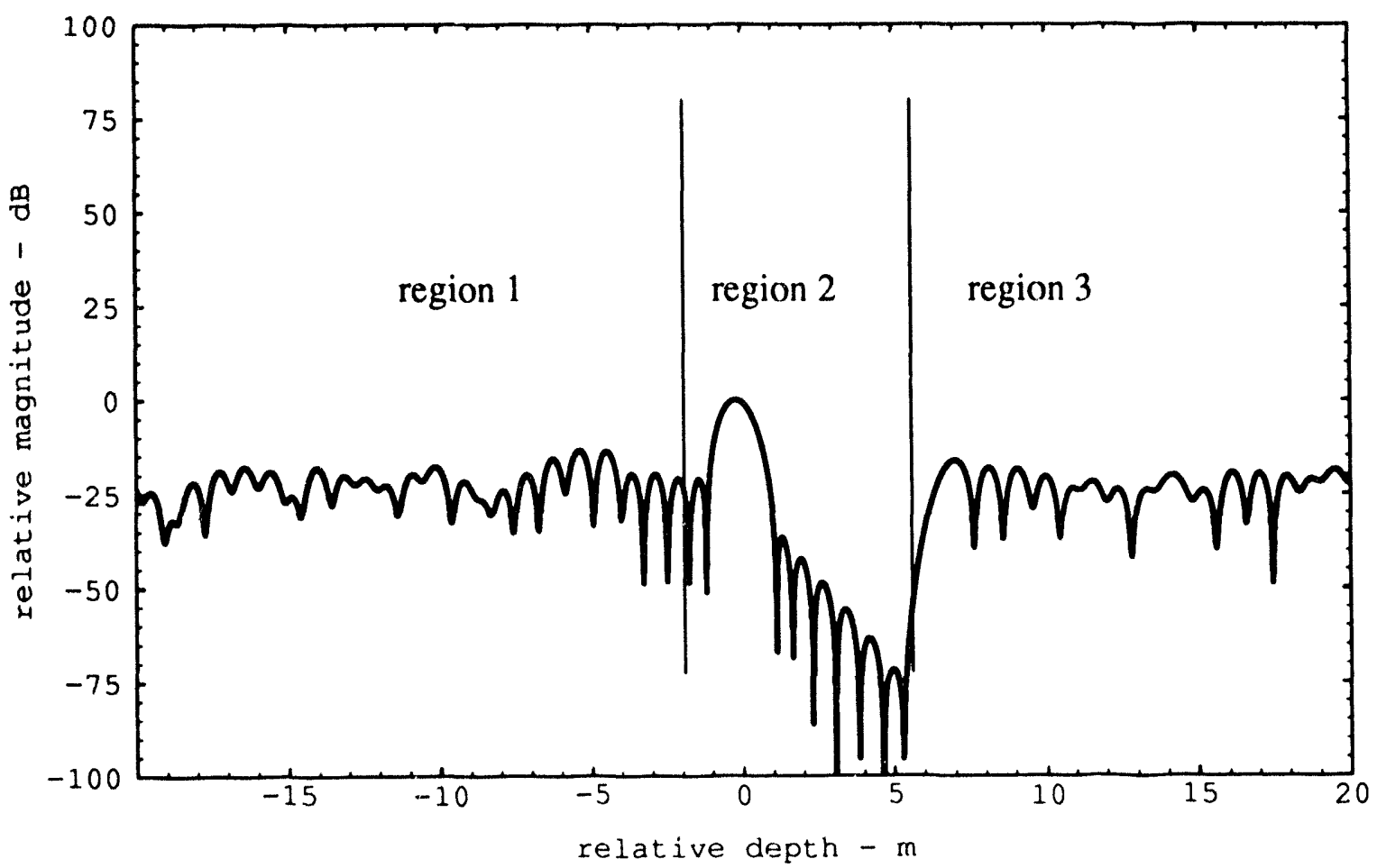

Figure 19. Characteristics of desired spectrum of window function. 


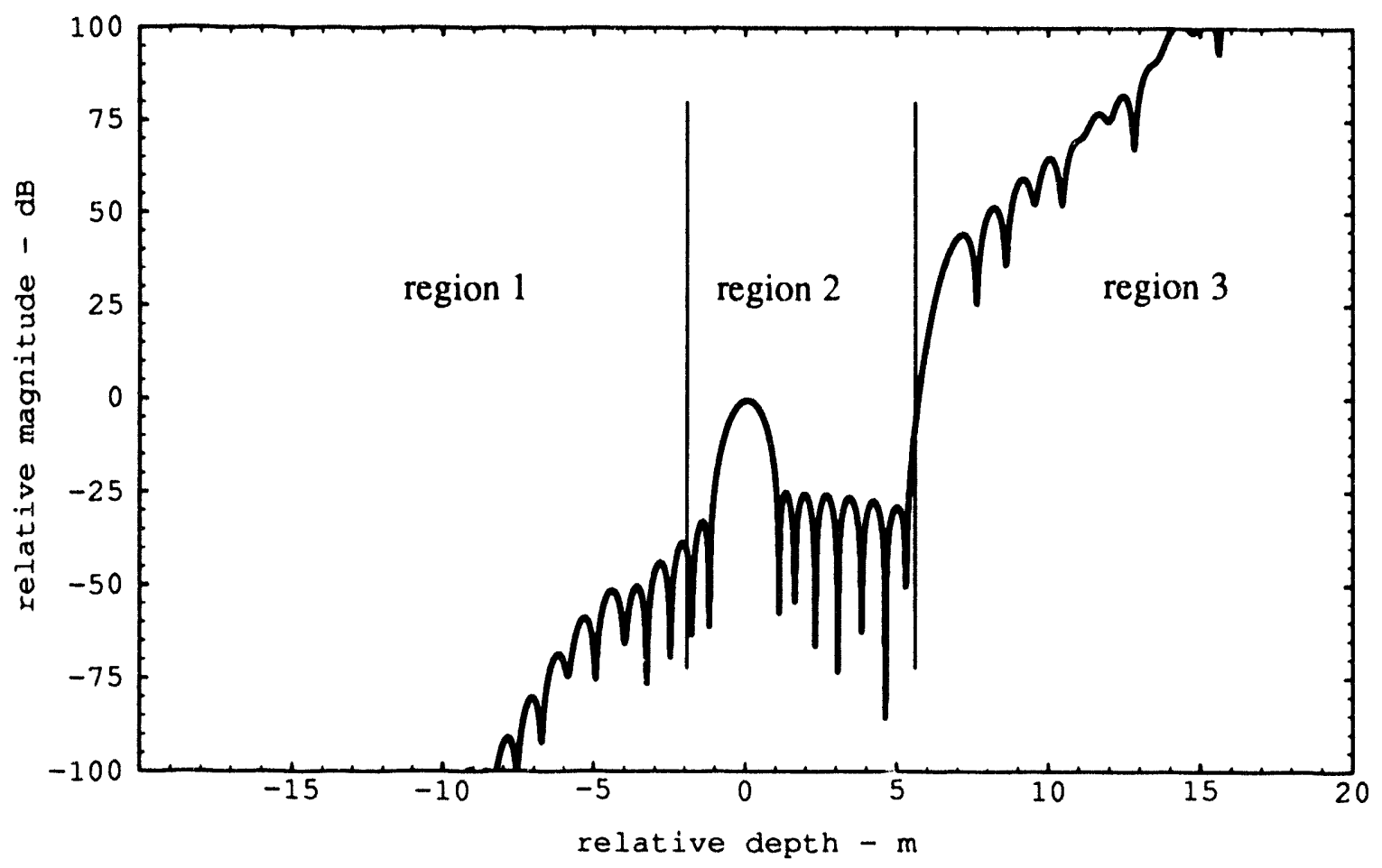

Figure 20. DFT-processed IPR with window of figure 19.

yield an IPR of the form in figure 20. As is shown, the window has compensated for the expected loss in region 2.

Some observations are in order.

- Note in figure 20 how the sidelobes in region 2 are all suppressed by at least $25 \mathrm{~dB}$ relative to the mainlobe, and how sidelobes in region 1 are suppressed by loss normalization. Sidelobes in region 3 will never appear above the depth horizon and are therefore inconsequential.

- The amplitude spectrum of the window used for figures 19 and 20 is not even. This implies that the window itself is complex.

- For the sidelobes to be flat in a loss normalized IPR, the nature of the loss normalization will need to be known and incorporated into the window function itself. In this sense, the window will need to be 'tuned' to, or compensated for the soil's lossiness.

- If region 2 is grown to extend to a deeper depth horizon, then the sidelobes in regions 1 and 3 will be stronger, and may even exceed the mainlobe. As the sidelobes grow and acquire more energy, the mainlobe will lose energy, resulting in a worsening SNR loss due to processing in the final image. This SNR loss will, in turn, tend to raise the depth horizon and diminish dynamic range.

We illustrate the benefits of loss compensated windows with an example. 
Example 9. Reconsider examples 6 and 8, where two targets were buried in soil with parameters that were representative of San Antonio Clay Loam with 5\% water at $300 \mathrm{MHz}$. We repeat the parameters here, and add a depth horizon of $3 \mathrm{~m}$.

$\begin{aligned} \text { relative dielectric constant } & 4.5-\mathrm{j} 1 \\ \text { relative permeability } & 1 \\ \text { depression angle } & 30 \text { degrees } \\ \text { center frequency } & 300 \mathrm{MHz} \\ \text { bandwidth } & 150 \mathrm{MHz} \\ \text { video samples (K) } & 128 \\ \text { target \#1 depth } & 0 \mathrm{~m} \text { (just below the surface) } \\ \text { target \#2 depth } & 2 \mathrm{~m} \\ \text { depth horizon } & 3 \mathrm{~m}\end{aligned}$

With a loss compensated window function applied prior to DFT processing (with loss normalization) we can achieve the result displayed in figure 21. Clearly, when the loss compensated window is used, the deeper target can be resolved. The slight offset in the mainlobe peaks is a compromise made to achieve a $3 \mathrm{~m}$ depth horizon in a $-28 \mathrm{~dB} / \mathrm{m}$ lossy soil.

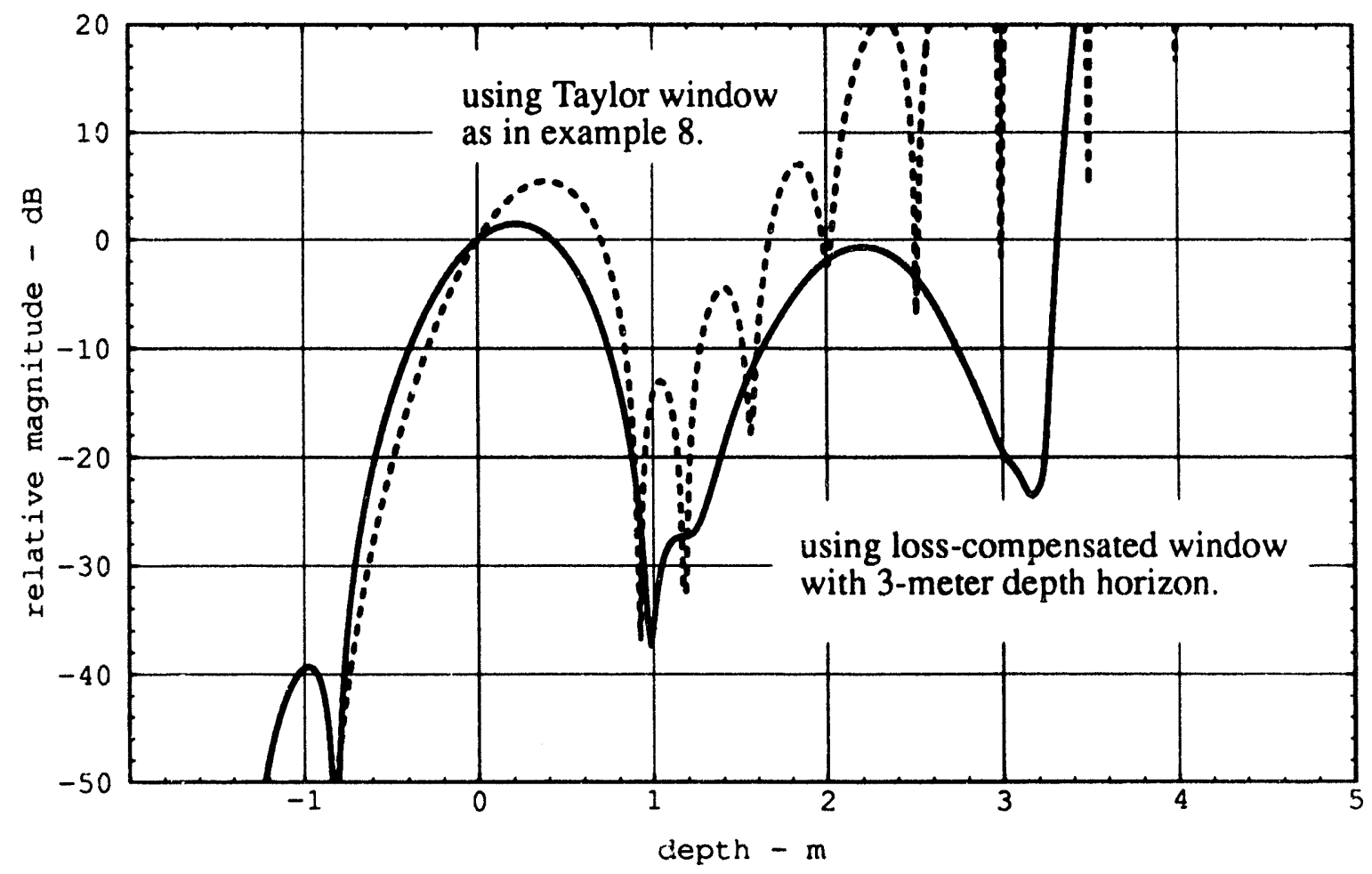

Figure 21. Effects of Loss Compensated Window Function on DFT-Processed IPRs. 


\subsection{Synthesizing Loss Compensated Windows}

The windows we are interested in are nothing more than finite impulse response (FIR) digital filters. The generation of loss compensated windows is an exercise in FiR digital filter synthesis, much as is described in reference [16], although in our case we are interested in complex filters. The tuned nature of the windows favor an iterative approach, where a filter is designed, tested, and redesigned until satisfactory results are obtained. The procedure used for this report finds a best mean-squared-error fit to a specification, and is as follows.

Specification. Select a set of normalized frequencies $f_{l}$, for $1 \leq l \leq L$, where $0 \leq f_{l}<1$. For each frequency $f_{l}$, specify a desired gain $d_{l}$, and a desired phase $\phi_{l}$. Also specify a desired cost $c_{l}$ that indicates the relative degree of desire for meeting the gain and phase specs. The greater $c_{l}$, the more critical the spec. As a practical matter, one can start with an existing window's spectral response and modify it in the region corresponding to region 2 of figure 19. The cost function is selected empirically, but a good initial guess is to begin with everything at $c_{l}=1$, and modify region 2 so that the cost is inversely proportional to the gain envelope. In addition, one might force the mainlobe peak to be nearly exactly the specified value by choosing its cost value very high, for example $10^{6}$. Let $n$ be the length of the desired window.

Calculation of Covariance Matrices. Calculate the auto-covariance and crosscovariance matrices as follows.

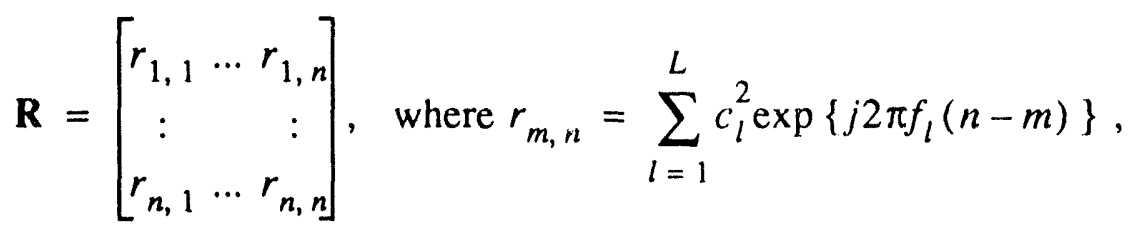

and

$\mathbf{P}=\left[\begin{array}{c}p_{1} \\ : \\ p_{n}\end{array}\right], \quad$ where $p_{n}=\sum_{l=1}^{L} d_{l} c_{l}^{2} \exp \left\{-j\left(2 \pi f_{l} n+\phi_{l}\right)\right\}$.

Calculate the Complex Window. Using the equations (33) and (34), the best, least-squared-error fit, window $\mathbf{w}^{*}$ can be calculated as follows.

$\mathbf{w}^{*}=\mathbf{a}+j \mathbf{b}$

where

$$
\begin{aligned}
& \mathbf{a}=\left(\mathbf{R}+\mathbf{R}^{T}\right)^{-1}\left[\left(\mathbf{P}+\mathbf{P}^{C}\right)+j\left(\mathbf{R}-\mathbf{R}^{T}\right) \mathbf{b}\right], \\
& \mathbf{b}=\left(\mathbf{R}+\mathbf{R}^{T}\right)^{-1}\left[j\left(\mathbf{P}-\mathbf{P}^{C}\right)-j\left(\mathbf{R}-\mathbf{R}^{T}\right) \mathbf{a}\right],
\end{aligned}
$$


and the superscripts ${ }^{T}$ and ${ }^{C}$ denote transpose and complex conjugate, respectively.

Equations (36) and (37) can be simultaneously solved. This allows us to calculate

$$
\begin{aligned}
\mathbf{b}=j\left[\left(\mathbf{R}+\mathbf{R}^{T}\right)-\left(\mathbf{R}-\mathbf{R}^{T}\right)\left(\mathbf{R}+\mathbf{R}^{T}\right)^{-1}\left(\mathbf{R}-\mathbf{R}^{T}\right)\right]^{-1} \\
\cdot\left[\left(\mathbf{P}-\mathbf{P}^{C}\right)-\left(\mathbf{R}-\mathbf{R}^{T}\right)\left(\mathbf{R}+\mathbf{R}^{T}\right)^{-1}\left(\mathbf{P}-\mathbf{P}^{C}\right)\right] .
\end{aligned}
$$

Combining equations (35), (36), and (38) yields the resulting window $\mathbf{w}^{*}$.

Reiteration. The spectrum of $\mathbf{w}^{*}$ can then be compared to the desired spectrum, and adjustments made to the specification. The most common specification change will likely be to adjust the depth horizon, and alter the cost specifications $c_{l}$. New calculations and comparisons are then in order.

The derivation of the best, least-squared-error fit, window $\mathbf{w}^{*}$ is given in Appendix B. 
THIS PAGE INTENTIONALLY BLANK 


\section{CONCLUSIONS}

This report touches a number of issues concerning imaging underground targets from an airborne radar. Conclusions in the form of observations are liberally sprinkled throughout the text of this report. Nevertheless, some more global conclusions can be reached and are presented here.

- A general closed form model of the radar echo is possible for an airborne radar imaging a target buried in a lossy half space, and is presented. Other approximations may at times also be adequate.

- For wideband systems, dispersion needs to be compensated, or at least accounted for.

- Attenuation for various subsurface media ranges from negligible to effectively opaque. In the $\mathrm{RF}$ region of the spectrum, attenuation generally increases with frequency.

- Synthetic Aperture Radar imaging relies on maintaining coherency in target echoes. This depends not only on sensor and target positions, but also on a reasonably accurate characterization of the RF signal's propagation. Errors in measured or assumed propagation parameters will manifest themselves in target position estimate inaccuracies, and image defocussing.

- Impulse responses should be thought of in terms of a 3-dimensional impulse response. Concepts like 'layover' easily fit into a 3-D IPR model.

- Resolution in 3 dimensions effectively requires a 2-dimensional synthetic spacial aperture along with a frequency aperture. How the spacial aperture is sampled will greatly impact sidelobe structure.

- Depth resolution is enhanced below the surface. Resolution in the horizontal directions is not.

- Soil lossiness requires a subsurface imaging radar to have larger dynamic range than its surface imaging counterpart. A dynamic range limit also limits penetration depth, and presents a 'depth horizon'.

- In a processed image, sidelobes of a shallow target in a lossy soil can easily overwhelm the response from a deeper target. These sidelobes can make a large dynamic range virtually useless. The sidelobes will be affected by system non-linearities and processing window functions. This may be mitigated somewhat with loss compensated window functions. But there are limits. 


\section{APPENDIX A}

\section{DERIVATION OF RADAR ECHO MODEL}

We begin with the geometry of figure 1 , and the exact echo delay time of equation (1) which is repeated here.

$$
\tau_{c}=\frac{2\left\|r_{c 1}\right\|}{c_{1}}+\frac{2\left\|r_{c 2}\right\|}{c} .
$$

The central assumption of this derivation is that $\psi_{2} \approx \psi$. This allows us to write

$$
\left\|r_{c 2}\right\| \approx\left\|r_{c}\right\|-\left\|r_{c 1}\right\| \cos \psi_{1} \cos \psi
$$

By recognizing that $\left\|r_{c 1}\right\|=\frac{d}{\sin \psi_{1}}$, and substituting (A2) into (A1), we arrive at the expression

$$
\tau_{c} \approx \frac{2}{c}\left(\left\|r_{c}\right\|+\frac{d}{\sin \psi_{1}}\left(\frac{6}{c_{1}}-\cos \psi_{1} \cos \psi\right)\right) \text {. }
$$

At this point we look to Balanis [1], borrow some notation, and define

$$
\begin{aligned}
& \gamma_{1}=\alpha_{1}+j \beta_{1}=j \frac{\omega}{c} \eta, \\
& \gamma_{2}=j \beta_{2}=j \frac{\omega}{c}, \\
& \sin \theta_{t}=\frac{\gamma_{2}}{\gamma_{1}} \cos \psi_{2}, \\
& q=\operatorname{Real}\left(\cos \theta_{t}\right) \beta_{1}+\operatorname{Imag}\left(\cos \theta_{t}\right) \alpha_{1}, \\
& t=\beta_{2} \cos \psi_{2} .
\end{aligned}
$$

Note that equation (A6) is Snell's Law for this geometry, but $\theta_{l}$ is complex due to the lossiness of the dielectric $\left(\alpha_{1} \neq 0\right)$.

Balanis shows that given $\omega, t$, and $q$,

$$
\begin{aligned}
& c_{1}=\frac{\omega}{\sqrt{t^{2}+q^{2}}}, \\
& \cos \psi_{1}=\frac{t}{\sqrt{t^{2}+q^{2}}}, \text { and }
\end{aligned}
$$




$$
\sin \psi_{1}=\frac{q}{\sqrt{t^{2}+q^{2}}} .
$$

Combining equations (A4) through (A8) into equations (A9) through (A11), and these in turn into equation (A3), yields after simplification the expression (recalling that $\psi_{2} \approx \psi$ )

$$
\tau_{c} \approx \frac{2}{c}\left(\left\|r_{c}\right\|+\operatorname{Real}\left[d \eta \sqrt{1-\left(\frac{\cos \psi}{\eta}\right)^{2}}\right]\right)
$$

The two-way power loss within the half space is given by the following attenuation factor

$$
G_{\eta}=\exp (-4 d \alpha)
$$

where, from Balanis,

$$
\alpha=\operatorname{Real}\left(\cos \theta_{t}\right) \alpha_{1}+\operatorname{Imag}\left(\cos \theta_{t}\right) \beta_{1}
$$

Using (A4) through (A6), and recalling that $\psi_{2} \approx \psi$, this can be rewritten as

$$
\alpha=-\frac{\omega}{c} \operatorname{Imag}\left[\eta \sqrt{1-\left(\frac{\cos \psi}{\eta}\right)^{2}}\right] .
$$

Combining with (A14) yields the final expression

$$
G_{\eta}=\exp \left(\frac{4 \omega}{c} \operatorname{Imag}\left[d \eta \sqrt{1-\left(\frac{\cos \psi}{\eta}\right)^{2}}\right]\right)
$$

At this point we can make several observations.

- The dependence of refraction angle and power loss within the lossy half space on dielectric properties is strictly via the complex refraction index, $\eta$. (This is not so for the loss associated with transmission across the dielectric boundary, which is essentially an impedance mismatch problem. See reference [1]).

- The echo delay time is linearly dependent on target depth, $d$.

- In general, $\eta$ is a function of frequency, $\omega$. In (A12) the resulting effect is known as dispersion. 


\section{APPENDIX B \\ DERIVATION OF OPTIMAL, COMPLEX FILTER}

Consider the following definitions.

$$
\begin{aligned}
& \mathbf{x}=\text { a column vector of input data, } \\
& \mathbf{w} \quad=\text { a column vector of filter (window) weights, } \\
& d \quad=\text { a scalar response. }
\end{aligned}
$$

We further identify

$$
\begin{aligned}
& \mathbf{x}=\left[\begin{array}{c}
\sum_{l=1}^{L} c_{l} \exp \left\{j 2 \pi f_{l}\left(\frac{t}{T}-n\right)\right\} \\
:
\end{array}\right], \\
& d=d_{l} c_{l} \exp \left\{j\left(2 \pi f_{l}\left(\frac{t}{T}\right)+\phi_{l}\right)\right\},
\end{aligned}
$$

where $\quad n=$ the vector element position index, and $0 \leq n \leq(N-1)$,

$$
\begin{aligned}
\mathrm{t} & =\text { time, } \\
\mathrm{T} & =\text { sampling interval, }
\end{aligned}
$$

and other parameters are as described in section 4.5.

We define the filter output as

$$
y=\mathbf{w}^{T} \mathbf{x}
$$

and the error in the output as

$$
\varepsilon=(d-y) .
$$

Recall that the superscripts ${ }^{T}$ and ${ }^{C}$ denote transpose and complex conjugate, respectively. We desire a minimum mean-squared-error (mse) and define mse as

$$
\mathrm{mse}=E\left[|\varepsilon|^{2}\right]=E\left[\varepsilon \varepsilon^{C}\right],
$$

where $E[\cdot]$ is the expected-value operator (over time).

Now for some linear algebra. 
We can readily expand

$$
|\varepsilon|^{2}=|d|^{2}+\mathbf{w}^{T} \mathbf{x} \mathbf{x}^{T C} \mathbf{w}^{C}-\mathbf{w}^{T} \mathbf{x} d^{C}-\mathbf{w}^{T C} \mathbf{x}^{C} d .
$$

Taking the expected value of equation (B6) yields

$$
\mathrm{mse}=E\left[|d|^{2}\right]+\mathbf{w}^{T} \mathbf{R} \mathbf{w}^{C}-\mathbf{w}^{T} \mathbf{P}-\mathbf{w}^{T C} \mathbf{P}^{C},
$$

where

$$
\mathbf{R} \equiv E\left[\mathbf{x x}^{T C}\right]
$$

and $\mathbf{P} \equiv E\left[\mathbf{x} d^{C}\right]$.

With $\mathbf{x}$ and $d$ defined as in (B1) and (B2), $\mathbf{R}$ and $\mathbf{P}$ become

$$
\mathbf{R}=\left[\begin{array}{ccc}
r_{1,1} & \cdots & r_{1, n} \\
: & & \\
r_{n, 1} & \cdots & r_{n, n}
\end{array}\right], \text { where } r_{m, n}=\sum_{l=1}^{L} c_{l}^{2} \exp \left\{j 2 \pi f_{l}(n-m)\right\},
$$

and

$$
\mathbf{P}=\left[\begin{array}{c}
p_{1} \\
: \\
p_{n}
\end{array}\right], \quad \text { where } p_{n}=\sum_{l=1}^{L} d_{l} c_{l}^{2} \exp \left\{-j\left(2 \pi f_{l} n+\phi_{l}\right)\right\}
$$

We now expand

$$
\mathbf{w}=\mathbf{a}+j \mathbf{b}
$$

where $\mathbf{a}$ and $\mathbf{b}$ are real.

Inserting (B12) into (B7) yields

$$
\begin{aligned}
\text { mse }=\left[E\left[|d|^{2}\right]+\mathbf{a}^{T}\right. & \left.\mathbf{R} \mathbf{a}^{C}+\mathbf{b}^{T} \mathbf{R} \mathbf{b}^{C}-\mathbf{a}^{T}\left(\mathbf{P}+\mathbf{P}^{C}\right)\right] \\
-j & {\left[\mathbf{a}^{T}\left(\mathbf{R}-\mathbf{R}^{T}\right) \mathbf{b}+\mathbf{b}^{T}\left(\mathbf{P}-\mathbf{P}^{C}\right)\right] }
\end{aligned}
$$


To find the optimal $\mathbf{a}$ and $\mathbf{b}$ that minimize the mse, we take the derivatives of mse with respect to $a$ and $\mathbf{b}$ and set them equal to zero.

$$
\frac{d}{d \mathbf{a}} m s e=\left(\mathbf{R}+\mathbf{R}^{T}\right) \mathbf{a}-j\left(\mathbf{R}-\mathbf{R}^{T}\right) \mathbf{b}-\left(\mathbf{P}+\mathbf{P}^{C}\right)=0,
$$

and

$$
\frac{d}{d \mathbf{b}} m s e=\left(\mathbf{R}+\mathbf{R}^{T}\right) \mathbf{b}-j\left(\mathbf{R}^{T}-\mathbf{R}\right) \mathbf{a}-j\left(\mathbf{P}-\mathbf{P}^{c}\right)=0 .
$$

This yields

$$
\begin{aligned}
& \mathbf{a}=\left(\mathbf{R}+\mathbf{R}^{T}\right)^{-1}\left[\left(\mathbf{P}+\mathbf{P}^{C}\right)+j\left(\mathbf{R}-\mathbf{R}^{T}\right) \mathbf{b}\right], \\
& \mathbf{b}=\left(\mathbf{R}+\mathbf{R}^{T}\right)^{-1}\left[j\left(\mathbf{P}-\mathbf{P}^{C}\right)-j\left(\mathbf{R}-\mathbf{R}^{T}\right) \mathbf{a}\right],
\end{aligned}
$$

These are equations (36) and (37) in the main text and may be solved simultaneously to yield equation (39) in the main text, repeated here as

$$
\begin{aligned}
\mathbf{b}=j\left[\left(\mathbf{R}+\mathbf{R}^{T}\right)-\left(\mathbf{R}-\mathbf{R}^{T}\right)\left(\mathbf{R}+\mathbf{R}^{T}\right)^{-1}\left(\mathbf{R}-\mathbf{R}^{T}\right)\right]^{-1} \\
\cdot\left[\left(\mathbf{P}-\mathbf{P}^{C}\right)-\left(\mathbf{R}-\mathbf{R}^{T}\right)\left(\mathbf{R}+\mathbf{R}^{T}\right)^{-1}\left(\mathbf{P}-\mathbf{P}^{C}\right)\right] .
\end{aligned}
$$

Substituting (B18) into (B16), and then both (B18) and (B16) into (B12) yields a unique expression for $\mathbf{w}$ in terms of only $\mathbf{R}$ and $\mathbf{P}$, that is the minimum mean-squared-error solution.

Note that for real $\mathbf{x}$ and real $d$, we have a real $\mathbf{R}$ and $\mathbf{P}$, and (B12) reduces to the more common expression $\mathbf{w}=\mathbf{R}^{-1} \mathbf{P}$. 
THIS PAGE INTENTIONALLY BLANK 


\section{REFERENCES}

[1] Balanis, Constatine A., “Advanced Engineering Electromagnetics”, ISBN 0-471-62194-3, John Wiley \& Sons, Inc., 1989.

[2] Brock, B. C., W. E. Patitz, "Optimum Frequency for Subsurface-Imaging SyntheticAperture Radar, Sandia National Laboratories Report SAND93-0815, May 1993.

[3] Campbell, Jeffrey E., "Dielectric Properties and Influence of Conductivity in Soils at One to Fifty Megahertz", Soil Sci. Soc., Am J., Vol. 54, p. 332, March-April 1990.

[4] Daniels, D. J., "Introduction to subsurface radar", IEE Proceedings, Vol. 135, Pt. F, No. 4, August 1988.

[5] Douglas, Dennis G., Alan A. Burns, Charles L. Rino, Joseph W. Maresca, Jr., “A Study to Determine the Feasibility of Using a Ground-Penetrating Radar for more Effective Remediation of Subsurface Contamination", Report EPA/600/R-92/089, PB92-169382, May 1992.

[6] Hansen, W., W. R. Sill, S. H. Ward, "The Dielectric Properties of Selected Basalts", Geophysics, Vol. 38, No. 1, pp. 135-139, February 1973.

[7] Hipp, Jackie E., "Soil Electromagnetic Parameters as Functions of Frequency, Soil Density, and Soil Moisture", Proceedings of the IEEE, Vol. 62, No. 1, January 1974.

18] Hoekstra, P., A. Delaney, "Dielectric Properties of Soils at UHF and Microwave Frequencies", Journal of Geophysical Research, Vol. 79, No. 11, p. 1699, April 10, 1974.

[9] Larson, Richard W., Jerry S. Zelenka, Elmer L. Johansen, “A Microwave Hologram Radar System", IEEE Transactions on Aerospace and Electronic Systems, Vol. AES-8, No. 2, p. 208, March 1972.

[10] Magotra, N., W. McCoy, B. K. Chang, "Narrow Band Synthetic Aperture Radar Image Formation”, ICASSP '91, Toronto, Ontario, Canada, p. 2609, May 14-17, 1991.

[11] Olhoeft, Gary R., "Processing, modelling, and presentation of ground penetrating radar data", Second Government Workshop on GPR, Fawcett Center, The Ohio State University, Columbus Ohio, 26-28 October 1993.

[12] Prati, C., F. Rocca, "Improving Slant-Range Resolution With Multiple SAR Surveys", IEEE Transactions on Aerospace and Electronic Systems, Vol. 29, No. 1, p. 135, January 1993.

[13] Scott, Waymond R. Jr., Glenn S. Smith, "Measured Electical Constitutive Parameters of Soil as Functions of Frequency and Moisture Content", IEEE Transactions on Geoscience and Remote Sensing, Vol. 30, No. 3, p. 621, May 1992. 
[14] Smith, D. V., "Propagation of Ground Penetrating Radar Signals in Soils", 2nd International Symposium on Geotechnical Applications of Ground-Penetrating Radar, University of Florida, Gainesville, Florida, 10 March 1988.

[15] Xu, Rongqing, Zhidao Cao, Fulin Su, "Synthetic Aperture Radar Imaging for Objects Imbedded in a Half Space Lossy Medium at Close Range", SPIE Vol. 1875 Ultrahigh Resolution Radar, 1993.

[16] Widrow, Bernard, Samuel D. Stearns, “Adaptive Signal Processing”, ISBN 0-13-004029 01, Prentice-Hall, Inc., Englewood Cliffs, New Jersey 07632, 1985. 
THIS PAGE INTENTIONALLY BLANK 


\section{DISTRIBUTION}

\begin{tabular}{|c|c|c|c|}
\hline copies & Mailstop & Dept. & Name \\
\hline 1 & MS 0509 & 2300 & R. D. Andreas \\
\hline 1 & MS 0531 & 2343 & W. H. Schaedla \\
\hline 1 & MS 0531 & 2343 & B. C. Brock \\
\hline 1 & MS 0531 & 2343 & W. E. Patitz \\
\hline 1 & MS 0531 & 2344 & R. M. Axline \\
\hline 1 & MS 0531 & 2344 & D. L. Bickel \\
\hline 1 & MS 0531 & 2344 & J. T. Cordaro \\
\hline 1 & MS 0531 & 2344 & W. H. Hensley \\
\hline 1 & MS 0529 & 2345 & B. C. Walker \\
\hline 1 & MS 0529 & 2345 & B. L. Burns \\
\hline 15 & MS 0529 & 2345 & A. W. Doerry \\
\hline 1 & MS 0529 & 2345 & D. F. Dubbert \\
\hline 1 & MS 0529 & 2345 & R. B. Hurley \\
\hline 1 & MS 0529 & 2345 & J. M. Opalka \\
\hline 1 & MS 0529 & 2346 & G. R. Sloan \\
\hline 1 & MS 0572 & 5912 & C. V. Jakowatz \\
\hline 1 & MS 0705 & 6114 & M. W. Scott \\
\hline 1 & MS 0304 & 9100 & C. M. Hart \\
\hline 1 & MS 0355 & 9131 & J. R. Fellerhoff \\
\hline 1 & MS 0355 & 9131 & D. H. Cress \\
\hline 1 & MS 0358 & 9135 & Bill Boverie \\
\hline 1 & MS 0355 & 9136 & F. M. Dickey \\
\hline 1 & MS 0985 & 2602 & M. W. Callahan \\
\hline 1 & MS 0105 & 9835 & T. J. Flynn \\
\hline 1 & MS 9018 & $8523-2$ & Central Technical Files \\
\hline 5 & MS 0899 & 7141 & Technical Library \\
\hline 1 & MS 0619 & 7151 & Technical Publications \\
\hline 10 & MS 0100 & $7613-2$ & Document Processing for DOE/OSTI \\
\hline 1 & D/852 MG39 & ASKC & C. T. Allen \\
\hline 1 & \multicolumn{3}{|c|}{$\begin{array}{l}\text { Dr. Neeraj Magotra } \\
\text { EECE Department } \\
\text { University of New Mexico } \\
\text { Albuquerque, NM } 87131-1356\end{array}$} \\
\hline
\end{tabular}



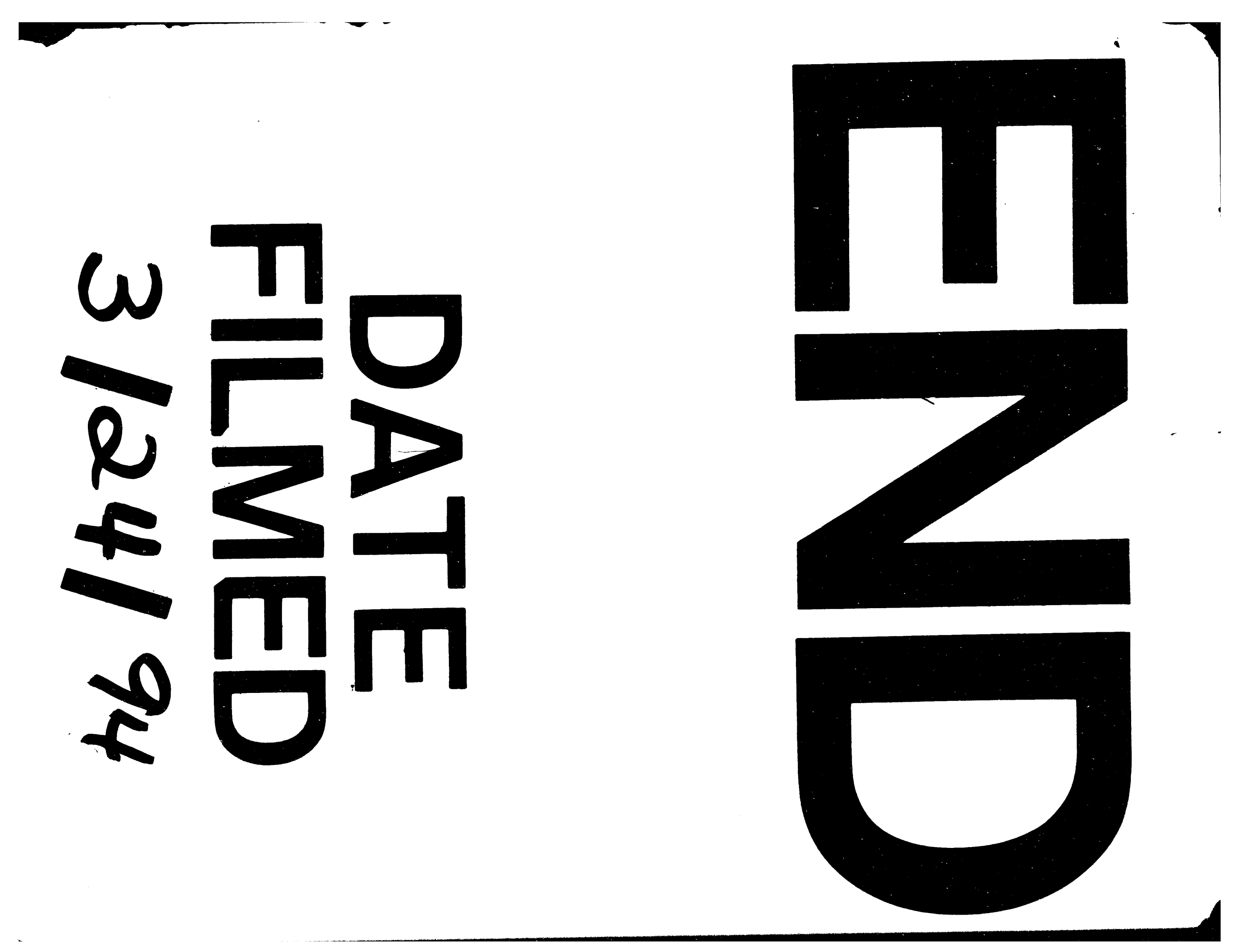
